\title{
¿Influyen los auditores en la agresividad fiscal de sus clientes? Evidencia empírica en las compañías españolas no cotizadas
}

\author{
Juan Monterrey Mayoral ${ }^{a}$, Amparo Sánchez Segura ${ }^{b}$ \\ a) b) Departamento de Economía Financiera y Contabilidad, Facultad de Ciencias Económicas y Empresariales, Universidad de Extremadura, Badajoz, SPAIN.
}

${ }^{a}$ Corresponding author.

E-mail address: jmontrey@unex.es

\section{A R T I C L E I N F O}

Article history:

Received 12 September 2019

Accepted 6 May 2020

Available online 1 January 2022

\section{Códigos JEL:}

Códig
M41

M42
H26

Palabras clave:

Auditoría

Contabilidad

Impuestos

\section{R E S U M E N}

Nuestro estudio tiene por objeto verificar empíricamente en qué medida los auditores influyen en las prácticas fiscales de sus clientes. Tomando una amplia muestra representativa de compañías españolas no cotizadas para el periodo comprendido entre 2009 y 2017, hemos documentado resultados empíricos que dejan constancia de que la elección de un auditor de calidad induce un significativo impacto en las estrategias de planificación fiscal de las empresas, que muestran una mayor agresividad tributaria cuando son auditadas por una Big Four. No obstante esta evidencia, el endeudamiento y la presencia de créditos fiscales pendientes de aprovechamiento se erigen como factores mitigantes de esta conducta. Además, la percepción por el auditor de un nivel de honorarios anormalmente elevados no parece generar incentivos para cooperar en las estrategias fiscales de la compañía auditada. Los resultados que hemos documentado son robustos y se mantienen inalterados tras corregir la posible endogeneidad inherente a la elección del auditor y ensayar con especificaciones alternativas de variables.

(C)2022 ASEPUC. Published by EDITUM - Universidad de Murcia. This is an open access article under the CC BY-NC-ND license (http://creativecommons.org/licenses/by-nc-nd/4.0/).

Auditors and tax aggressiveness: Empirical evidence in private Spanish companies

A B S T R A C T

The purpose of our study was to carry out an empirical test of the extent to which auditors have an influence on the tax practices of the audited firm. Based on a wide sample of Spanish non-listed companies for the period 2009-2017, we have obtained consistent empirical evidence revealing that the choice of a high-quality auditor has a significant impact on the tax planning strategy of the firm. Companies show a greater tax planning aggressiveness when they are audited by one of the Big Four. Notwithstanding, leverage and the existence of tax credits are factors mitigating that aggressiveness. Moreover, abnormally higher audit fees do not seem to be an incentive for the auditor to cooperate in the tax strategies of the audited company. Our results are robust and remain unaltered after adjusting for the potential heterogeneity inherent to auditor's choice and using alternative variable specifications.

(C)2022 ASEPUC. Publicado por EDITUM - Universidad de Murcia. Este es un artículo Open Access bajo la licencia CC BY-NC-ND (http://creativecommons.org/licenses/by-nc-nd/4.0/). 


\section{Introducción}

El presente trabajo tiene por objeto analizar la influencia de la calidad de los auditores en la conducta tributaria de sus clientes, cuestión que en los últimos años ha sido objeto de amplio debate, tanto en el ámbito de los impuestos, en el que las estrategias de planificación fiscal agresivas son objeto de debate público, y el contexto de la auditoría, en el que la controversia sobre la independencia del auditor se mantiene abierta. Por ambas razones, consideramos que nuestro estudio reviste interés, situándose en la intersección entre las prácticas fiscales y la posición de los auditores, y por ello nuestros resultados podrían contribuir al entendimiento de este, a nuestro juicio, interesante debate.

La literatura empírica sobre impuestos ha documentado de manera precisa y constante las notables consecuencias que las prácticas de planificación fiscal proyectan sobre cuestiones tan importantes como la rentabilidad presente y futura de las compañías (Lev \& Nissim, 2004), los precios y rentabilidades bursátiles (Hanlon \& Slemrod, 2009), la calidad del resultado (Hanlon, 2003), la estructura financiera (Graham \& Tucker, 2006), el coste de capital (Cook et al., 2017), las políticas de inversión (Berger, 1993) e incluso inciden en cuestiones tan estratégicas como la propia localización de las empresas (Altshuler et al., 2000) o las modificaciones estructurales del calado de fusiones, adquisiciones y escisiones (Erickson \& Wang, 2007).

Sin embargo, según nuestro parecer aún es insuficiente la literatura que documenta qué características y atributos empresariales son los que inducen dichas estrategias de planificación, más allá de la estructura de propiedad (Badertscher et al., 2013; Chen et al., 2010), la compensación salarial de los directivos (Desai et al., 2007) o las prácticas de gobierno corporativo (Armstrong et al., 2015; Monterrey \& SánchezSegura, 2015), y sorprende la escasez de estudios que abordan la función que desempeñan los auditores en las actividades de planificación fiscal de sus clientes. Hasta donde nuestro conocimiento alcanza, los de Kanagaretnam et al. (2016), Klassen et al. (2015), McGuire et al. (2012) e indirectamente los de Chen et al. (2019) y Gaaya et al. (2017) constituyen el reducido núcleo de estudios que solo muy recientemente han abordado esta cuestión.

Por esta razón nos parece oportuno llevar a cabo un estudio empírico con el objeto de verificar si, en el caso español, el auditor puede influir en las estrategias tributarias de su cliente, y en caso de que así sea, si dicha influencia se erige como un factor coadyuvante de su posible agresividad fiscal o, por el contrario, cumple un papel mitigante. Aunque es cierto que la función principal del auditor consiste en expresar una opinión acerca de la imagen fiel de las cuentas anuales de la entidad auditada, no lo es menos que en el curso de su trabajo, y como parte esencial de éste, analiza, obtiene evidencia, evalúa y concluye sobre la validez y razonabilidad de todos los aspectos fiscales que guardan relación con dichas cuentas anuales, incluidos los riesgos inherentes a ellos. Y así, como consecuencia de poseer un conocimiento detallado de la práctica fiscal de su cliente, el auditor puede mantenerse neutral o, por el contrario, adoptar una posición activa e influir en su conducta tributaria.

Conviene destacar que en España, la Ley 22/2015, de 20 de julio, de Auditoría de Cuentas, no prohíbe de manera expresa la prestación de servicios de asesoramiento tributario por parte del auditor, salvo en aquellos casos en los que tal desempeño pudiera afectar a su independencia. La Sentencia del Tribunal Supremo de 26 de febrero de 2018 consideró que "no parece razonable considerar incompatible la prestación de servicios de auditoría con el asesoramiento fiscal, de modo general", salvo en aquellos casos en los que dicha prestación pudiera afectar a la independencia del auditor. Así pues, ha de entenderse que la compatibilidad es la regla y la incompatibilidad la excepción.

Por otra parte, también nos parece conveniente precisar que la planificación fiscal no debe entenderse como una conducta inapropiada, sino como una actividad legítima, que forma parte integral de la estrategia empresarial y que consiste en aplicar la normativa identificando las posibilidades que ésta brinda para reducir o diferir la carga tributaria, generalmente beneficiándose de las economías de opción disponibles, y que han de diferenciarse de prácticas dudosas o incluso frontalmente contrarias a la norma materializadas recurriendo a operaciones simuladas o sin fondo económico real. Así pues, según nuestro parecer la planificación fiscal ha de aceptarse como una función empresarial más, de la que surgen decisiones en las que se ponderan tanto los beneficios inherentes a esta actividad como los costes y riesgos a ella asociados.

Nuestro trabajo se suma a la muy incipiente literatura que examina la relación del auditor con la práctica tributaria de sus clientes y documenta una primera evidencia empírica para el caso de las empresas no cotizadas españolas, dejando constancia de que la elección de un auditor de calidad favorece las estrategias de planificación fiscal; evidencia que se ve condicionada por nuestro entorno institucional, tanto de la auditoría de cuentas como de la fiscalidad empresarial. Y es en esta confluencia entre auditoría e impuestos en la que deben enmarcarse los resultados que presentaremos y discutiremos a lo largo del trabajo.

El resto del trabajo se organiza como sigue. En la segunda sección efectuamos una exposición de nuestros argumentos teóricos y revisamos la literatura sobre la materia; en la tercera presentamos la muestra de emoresas, la caracterización de las variables y la especificación econométrica del modeño diseñado para el análisis empírico; en la sección cuarta mostramos y discutimos los resultados empíricos principales; en la sección quinta llevamos a cabo algunas extensiones y pruebas adicionales para profundizar en la evidencia obtenida y garantizar su robustez, y en la sección sexta concluimos y señalamos tanto las limitaciones que hemos identificado en el estudio como posibles vías futuras de investigación.

\section{Argumentos teóricos y evidencia empírica previa}

\subsection{Argumentos teóricos y planteamiento de hipótesis: incen- tivos económicos y protección frente al riesgo del auditor}

Como antes hemos afirmado, aunque la principal función del auditor consiste en expresar su opinión acerca de la imagen fiel de las cuentas anuales de su cliente, en el curso de su trabajo debe obtener evidencia suficiente y adecuada, efectuar las oportunas pruebas sustantivas y de cumplimiento y concluir con su parecer sobre la razonabilidad de los estados financieros en su conjunto. Entre las áreas que el auditor debe verificar se encuentra, y diríamos que de manera prominente, la relativa a los impuestos, en la que se requiere analizar la recuperabilidad de los créditos fiscales, la generación y reversión de las diferencias temporarias imponibles y deducibles, la corrección de las declaraciones tributarias, la delimitación, en su caso, del grupo de consolidación fiscal, la aplicación de deducciones y ventajas fiscales y, en fin, calibrar los riesgos de naturaleza tributaria a efectos de valorar las provisiones y contingencias de esta naturaleza. 
Como podemos apreciar, se trata de una tarea que incluye diferentes funciones y que, además, es siempre material, tanto en el balance como en la cuenta de resultados y en la información contenida en la memoria, y que requiere que el auditor posea un elevado nivel de conocimiento técnico. Asimismo, tiene que evaluar el grado de agresividad fiscal de su cliente y si está o no implicado en transacciones con potenciales riesgos tributarios, por lo que, llegado el caso, puede recomendar o requerir a su cliente modificaciones sustantivas en algunas de sus prácticas, como, por ejemplo, la valoración de transacciones con partes vinculadas ${ }^{1}$, las provisiones para impuestos, el reconocimiento o la baja de créditos fiscales, las diferencias temporarias, etc.

Por todo ello, el auditor tiene un importante papel que desempeñar en las materias tributarias de su cliente, y de aquí que su propia actitud frente a estas prácticas pudiera erigirse como uno de los elementos determinantes de la conducta fiscal de quien le contrata. Ahora bien, la cuestión central consiste en conocer cual es la posición del auditor frente a la agresividad fiscal de su cliente, para lo que puede adoptar tres actitudes distintas: mantenerse neutral, cooperar en las actividades de planificación fiscal para reducir o diferir la carga tributaria o, por último, impedir estrategias agresivas que, al exacerbar los riesgos de su cliente, se conviertan en riesgos del propio auditor. Como hemos visto, la escasa literatura existente sobre la materia aporta resultados empíricos divergentes entre sí, y que varían en función del contexto, de la situación y del entorno institucional del auditor.

Según nuestro parecer, la implicación o no del auditor en los asuntos tributarios del cliente, y si dicha implicación se orienta en un sentido cooperador o mitigador de su agresividad fiscal, sería el resultado final de un análisis en el que se ponderasen dos elementos que actúan en sentido contrario: de una parte, los incentivos económicos inherentes a ampliar su oferta de servicios y, como consecuencia de proporcionarle esta mayor cobertura, reforzar la fidelización de su cliente. De otra, la duda de si con ello quedaría dañada su independencia, tanto por el inevitable hecho de estrechar sus vínculos como por aumentar la dependencia económica con la empresa, lo que finalmente podría afectar a la calidad de su trabajo como auditor (DeFond \& Zhang, 2014), y, asimismo, el riesgo de verse implicado en litigios y procedimientos cuya visibilidad podría erosionar su capital reputacional. Si los incentivos económicos dominan sobre las amenazas a la calidad de la auditoría y a los riesgos reputacionales, el auditor se orientará a la cooperación con el cliente en sus asuntos fiscales, y sucederá lo contrario en la medida en que el deseo de evitar riesgos le haga descartar cualquier colaboración con prácticas fiscales agresivas.

Una primera posibilidad es que el auditor coadyuve con el cliente en el diseño de sus estrategias fiscales, lo que podría explicarse si, a medida que va profundizando en el conocimiento de su cliente, considera que ello reduce su propio propio riesgo, aumentando de este modo los incentivos existentes para ampliar su oferta de servicios y maximizar las rentas obtenidas. Así, está claramente documentado que cuando el contrato con su cliente se prolonga por varios ejercicios, el auditor va profundizando en las circunstancias de éste, adquiere conocimientos específicos --entre los que se encuentran los de naturaleza tributaria--, y, como muestran Bowlin et al. (2015) y Myers et al. (2003), la curva de aprendizaje le permite reducir la probabilidad de incurrir en errores y

${ }^{1}$ El estudio de Klassen et al. (2017), referido al caso norteamericano, demuestra que las operaciones con partes vinculadas constituyen el recurso más empleado por las empresas que muestran los niveles más elevados de agresividad fiscal. mitigar toda clase de riesgos, y de aquí que el auditor emplearía su conocimiento del cliente para implicarse en cuestiones impositivas en beneficio de éste. Desde esta perspectiva, el auditor aceptaría un determinado nivel tolerable de agresividad fiscal y, en contrapartida, asumiría un nivel también aceptable de riesgo.

La segunda posibilidad es que el auditor sea reacio a implicarse en estrategias fiscales agresivas que generen riesgos adicionales a los que ya asume en su trabajo de auditoría. Desde este punto de vista, los auditores a los que se les reconocen las mayores credenciales profesionales tendrían menores incentivos para verse envueltos en este tipo de prácticas, de manera que los incentivos pecuniarios serían insuficientes para compensar cualquier riesgo asumido y ocasionar un daño a su capital reputacional, que de este modo quedaría protegido. Así, Donohoe \& Knechel (2014) sugieren que las empresas fiscalmente agresivas exponen a sus auditores a un mayor riesgo de litigios, en la medida en que sustentar este tipo de posiciones puede llevar a los accionistas a invocar que el auditor es un cooperador en el fraude fiscal, o, al menos, en exacerbar este riesgo, y Hanlon \& Slemrod (2009) y Kim et al. (2011) aportan evidencia de la adversa reacción del mercado ante la aparición de escándalos tributarios, lo que, siguiendo la estela de su cliente, podría dañar la reputación del auditor.

También está bien descrito en la literatura cómo las posiciones fiscales agresivas dañan la calidad de la información contable, en la medida en que estas prácticas a menudo emplean como recurso la dirección de resultados (Frank et al., 2009; Phillips et al., 2003; Schrand \& Wong 2003). Así pues, si la agresividad fiscal conlleva una mayor agresividad contable, y, en consecuencia, ello va en detrimento de la calidad de la información financiera, el riesgo del auditor se vería amplificado, de modo que su interés por mitigarlo le llevaría a no implicarse en tales prácticas.

Así pues, el efecto que el auditor pueda ejercer sobre las prácticas fiscales de su cliente es una cuestión abierta y por ello requiere la oportuna verificación empírica, y de aquí que la hipótesis nula objeto de contraste sea la que formulamos seguidamente:

\section{$H_{o}$ : Las empresas que contratan un auditor de cali- dad no exhiben una agresividad fiscal diferente a las empresas auditadas por otras firmas.}

Con la finalidad de someter a contraste empírico la hipótesis nula que acabamos de enunciar, llevaremos a cabo la regresión del modelo cuya expresión genérica es la siguiente:

$$
P F_{i t}=\alpha+\beta B 4_{i t}+\delta X_{i t}+\text { Años }+ \text { Sectores }+\varepsilon_{i t}
$$

En el que, para cada firma $i$ y ejercicio $t$, la variable dependiente, $P F_{i t}$, es una caracterización de la agresividad fiscal, de la que seguidamente nos ocuparemos; $B 4_{i t}$ es la variable experimental, que se expresa en términos binarios y que toma el valor 1 si las cuentas anuales son verificadas por una de las cuatro grandes firmas de auditoría y cero en caso contrario, y $X_{i t}$ es un vector de variables de control que capta las características de las empresas que la literatura ha documentado como potenciales determinantes de las estrategias de planificación fiscal. Además, el modelo incorpora dos controles adicionales, uno temporal y otro de filiación sectorial.

En cuanto a la variable experimental, $B 4_{i t}$, es el subrogado que emplearemos de la calidad del auditor. Las cuatro grandes firmas no solo acreditan elevados estándares de calidad como auditores, sino que, además, poseen probada experiencia y capacidad en materia fiscal, y de aquí que a su tarea 
como revisores puedan añadir elevadas prestaciones en materia impositiva, siendo una cuestión empírica, como hemos avanzado, en qué dirección aplican su caudal de conocimientos tributarios. Desde el trabajo seminal de DeAngelo (1981) se acepta de manera unánime que el tamaño del auditor es una representación apropiada de su calidad, ya que caracteriza tanto su capital reputacional como su independencia económica del cliente y su capacidad técnica. Si en nuestro posterior análisis empírico el coeficiente $\beta$ asociado a $B 4_{i t}$ resulta ser estadísticamente significativo, dejaría constancia de que el hecho de contratar a un auditor Big Four también afecta a la práctica fiscal, y en el caso de no alcanzar los niveles convencionales de significación estaría indicando que las cuatro grandes firmas de auditoría no ejercen efectos sensibles sobre las actividades tributarias de sus clientes.

\subsection{Evidencia empírica previa documentada en la literatura}

Como hemos avanzado al comienzo de este trabajo, la literatura que relaciona la calidad de auditoría con las prácticas tributarias es tan reciente como reducida, estando confinada a un pequeño grupo de estudios. Podemos señalar el de McGuire et al., (2012) como el primero de los trabajos dentro de esta línea de investigación, y en que los autores seleccionan una muestra de empresas cotizadas en las que éstas reciben de su auditor, además del servicio de verificación de sus estados financieros, asesoramiento fiscal, documentando cómo las empresas que contratan auditores que cuentan con divisiones altamente especializadas en materias impositivas -entendiendo por tales las que tienen una participación superior al $25 \%$ en su mercado local de consultoría tributaria-muestran una mayor agresividad fiscal que aquellas otras cuyo auditor no posee experiencia en este campo, concluyendo que la acreditación del auditor en el ámbito tributario juega un papel significativo en las estrategias fiscales de su cliente, proporcionándole así un valor añadido adicional.

Klassen et al. (2015) parten de asumir que, por la naturaleza y características de su trabajo, el auditor es también responsable de la calidad de las prácticas tributarias de su cliente, según ellos al mismo nivel que los asesores fiscales de las compañías o que su propio departamento de impuestos. Bajo esta premisa, tomando una muestra de empresas cotizadas y empleando datos tributarios confidenciales de la Administración fiscal norteamericana (Internal Revenue Service), alcanzan como conclusión, entre otras, que los clientes asesorados en materia tributaria por las cuatro grandes firmas de auditoría, las Big Four, muestran un mayor conservadurismo fiscal que las que reciben este mismo servicio de otros asesores, y este mayor conservadurismo se observa en todos sus clientes, tanto en los que también se presta el servicio de auditoría como en aquellos que son auditados por otras firmas. Esta evidencia constituiría una manifestación de la preocupación de las Big Four por evitar riesgos que puedan afectar a su reputación.

Gaaya et al. (2017) examinan las actividades de planificación fiscal en las compañías cotizadas en Túnez de propiedad familiar, y sus resultados dejan constancia de que, si bien la agresividad es más acusada en las empresas familiares que en las de otro tipo de propiedad, la presencia de un auditor de calidad mitiga esta conducta y modera los incentivos de las empresas familiares para implicarse en estrategias de reducción de la presión fiscal.

El muy reciente estudio de Chen et al. (2019), referido a empresas no cotizadas del Reino Unido y, por sus características, próximo al nuestro, arroja resultados que indican que las empresas auditadas por las Big Four evitan el pago de impuestos en mayor medida que los clientes de los restantes auditores. En opinión de los autores, ello se debe a que en el segmento de las empresas sin cotización, las Big Four otorgarían prioridad a la eficiencia de las prácticas fiscales de sus clientes sobre la calidad de su información contable, lo que, según su parecer, sería una manifestación de que en un entorno de menor visibilidad, como es el de las empresas no cotizadas y en el que los objetivos de calidad contable del cliente serían de segundo orden con relación a los de carácter tributario, la percepción de riesgo reputacional de las Big Four es muy diferente al que se contrae en el contexto del mercado de capitales, lo que explicaría la mayor cooperación de las firmas de auditoría con las estrategias fiscales agresivas de sus clientes.

El trabajo de Kanagaretnam et al. (2016) aborda el análisis empírico de la relación entre calidad de auditoría y planificación fiscal en el plano internacional y es el único de los estudios específicamente dedicados al estudio de la influencia del auditor en la planificación fiscal que muestra una relación negativa de la calidad de auditoría con la agresividad tributaria. Para ello toman una muestra de empresas cotizadas pertenecientes a 31 países y seleccionan como subrogado de la calidad del auditor la pertenencia de éste al grupo de las Big Four. Tras estratificar su muestra de empresas, toman aquellas observaciones de cada país que se encuentran situadas en el primer quintil de agresividad fiscal y obtienen evidencia de una relación negativa con la calidad de auditoría, incluso después de controlar factores institucionales como las características del sistema fiscal de cada país.

Asimismo, Kanagaretnam et al. (2016) documentan cómo la relación negativa entre calidad del auditor y agresividad es más pronunciada en países de fuerte protección al inversor, elevado riesgo de litigios para el auditor, mejor entorno de auditoría y mayor presión de los mercados de capitales. Según nuestro parecer, los resultados de este estudio, contradictorios, como decimos, con la evidencia generalmente observada, podrían estar afectados por tres circunstancias; la primera, por la medida de agresividad fiscal seleccionada, inédita en la literatura, al tratarse de la diferencia entre el impuesto estatutario devengado y el impuesto corriente pagado (excluyendo su componente diferido); la segunda, por confinar el objeto de estudio a un segmento muy reducido de empresas, las más agresivas fiscalmente, lo que podría explicar la no cooperación del auditor, en este subconjunto de empresas, en su deseo de evitar riesgos, y en tercer lugar, por no experimentar simultáneamente con varias medidas empíricas de agresividad, que es el protocolo habitual en esta línea de investigación.

Por último, existen otros estudios que, aunque de manera expresa no analizan la relación entre calidad de auditoría y planificación fiscal, también aportan evidencias indirectas de ello, al incluir en sus modelos empíricos sobre planificación fiscal una variable dicotómica representativa de un auditor de calidad. Así, Hoopes et al. (2012) y Rezaee (2005) documentan cómo las Big Four protegen firmemente su reputación y no cooperan con la práctica fiscal de sus clientes, mitigando la agresividad de éstos al mostrarse una relación positiva entre las características del auditor y la presión fiscal. No obstante, autores como Badertscher et al. (2013), Lisowsky (2010) y Richardson et al. (2013) obtienen resultados que revelan una relación negativa entre las Big Four y la carga tributaria soportada, lo que, en opinión de ellos, obedecería a que las cuatro grandes firmas tienen recursos y capacidad para proporcionar a sus clientes soluciones a problemas fiscales de elevada complejidad técnica. En cualquier caso, los resultados de estos estudios que muestran una evidencia empírica 
indirecta han de ser tomados con suma cautela, ya que por no estar orientados específicamente al estudio de la relación entre calidad de auditoría y agresividad fiscal pueden presentar sesgos como consecuencia de las diferentes interacciones entre las variables empleadas.

En suma, la evidencia recogida en la literatura se orienta mayoritariamente en la dirección que apunta a la cooperación del auditor con las prácticas de planificación fiscal de su cliente, si bien con alguna excepción, señaladamente la de Kanagaretnam et al. (2016), cuyos resultados son, en nuestra opinión, cuestionables, como ya hemos comentado. En cualquier caso, los resultados empíricos documentados en estos trabajos no son ajenos al proglema generalmente planteado en esta línea de investigación, como es su sensibilidad a la metodología empleada y a las diferentes medidas de agresividad. Lo que sí parece unánime, a tenor de lo comentado, es que el auditor no es neutral ni impasible ante las circunstancias tributarias de sus clientes.

\section{Muestra y variables}

\subsection{Extracción de la muestra de empresas}

Dado que por razones de confidencialidad no es posible acceder a los microdatos procedentes de las declaraciones tributarias de las empresas, el análisis empírico necesariamente ha de desplegarse tomando como base la información procedente de las cuentas anuales de las empresas, y por esta razón las mediciones tanto del book-tax gap como de los tipos impositivos efectivos son imperfectas, lo que en la investigación empírica sobre impuestos se acepta como un hecho inevitable (Hanlon 2003; Blouin 2014). Así las cosas, nuestra muestra de empresas ha sido extraida de la base de datos $\mathrm{Sabi}^{\circledR} \mathrm{y}$ abarca, para los nueve ejercicios comprendidos entre 2009 y 2017, ambos inclusive, las sociedades mercantiles domiciliadas en España, activas, auditadas, que formulan cuentas anuales normales individuales y pertenecen a todos los sectores económicos excepto financiero y bancario (CNAE 64), seguros (CNAE 65) y los CNAE 84 y 99, por desarrollar actividades cuyas peculiaridades aconsejan excluirlas de este trabajo.

A los efectos de mitigar un posible sesgo de supervivencia que pudiera afectar a nuestros resultados empíricos, hemos formado una muestra variable, de manera que está integrada por todas las empresas presentes en $\mathrm{Sabi}^{\circledR}$ que en un determinado ejercicio cuentan con suficientes datos (seis ejercicios consecutivos) para configurar las variables requeridas. Tras eliminar de la muestra inicial las empresas que presentan discontinuidad en los datos o éstos son ilegibles o inexistentes, la muestra definitiva contiene 30.371 observaciones empresas-años, de la que no se han suprimido, como es habitual en la literatura empírica sobre planificación fiscal --con la excepción de Desai \& Dharmapala (2006)-- las observaciones influyentes, ya que su eliminación, como sostienen Armstrong et al. (2015), nos impediría conocer el impacto de las estrategias fiscales más agresivas y más conservadoras.

El hecho de configurar la muestra con cuentas individuales y no consolidadas tiene por finalidad asegurar que todas las compañías que la integran están sujetas al mismo marco normativo y a los mismos tipos nominales del impuesto, ya que una muestra formada a nivel de grupos consolidados integraría entidades con filiales que, al estar algunas de ellas domiciliadas en otros países, no son sujetos pasivos del impuesto en España, sino en otras jurisdicciones con diferentes regulaciones y tipos impositivos, lo que podría conducir a estimaciones erróneas tanto de las bases imponibles como de las diferencias discrecionales, y con ello a confundir nuestros resultados empíricos.

Además, hemos requerido que todas las observaciones presentes en la muestra tengan resultados antes de impuestos positivos, ya que, como bien razona Fernández Rodríguez (2004b), cuando éste es negativo los tipos efectivos calculados son una representación errónea de la presión fiscal realmente soportada: si tanto el resultado antes de impuestos como el pago por impuesto son negativos, el tipo efectivo resultante resulta ser positivo, pero en realidad la empresa no ha soportado ninguna presión fiscal, y si el resultado antes de impuestos es negativo y el pago por impuesto es positivo, la empresa soporta presión fiscal pero el tipo efectivo que se obtiene es negativo.

Aunque algunas de las variables que emplearemos en el análisis empírico, como los pagos del Impuesto sobre Sociedades y activos y pasivos por impuestos diferidos, ya estaban disponibles desde 2008, por tratarse del primer ejercicio en el que las empresas españolas formularon sus cuentas anuales con base en el vigente Plan General de Contabilidad, la muestra se inicia en 2009 como consecuencia de que las variables representativas de los impuestos diferidos, $A I D_{i t-1}$ y $P I D_{i t-1}$, así como $B T G_{i t-1}$, se expresan con un retardo de un año. También, dada la longitud de nuestra serie temporal, podría ocasionarse un cierto sesgo de supervivencia, y para mitigar su posible efecto hemos permitido que las compañías puedan entrar y salir de la muestra, configurando una muestra variable integrada por todas las empresas que, reuniendo todas las características antes indicadas, cuentan con suficientes datos en un determinado ejercicio que permitan disponer de las variables necesarias.

No obstante, la literatura sobre planificación fiscal coincide en apuntar que las prácticas tributarias se caracterizan de un modo más apropiado si la muestra excluye aquellas observaciones en las que el tipo impositivo efectivo oscila entre 0 y 1 , ya que los valores fuera de este rango plantean problemas de interpretación (Armstrong et al., 2015; Badertscher et al., 2013; Davis et al., 2016; Gallemore \& Labro 2015). Además, como demuestran empíricamente De Simone et al. (2020), los resultados ganan en robustez si en la muestra se excluyen las observaciones que caen fuera del citado rango (65 observaciones). Tras su eliminación, la muestra final de trabajo quedó configurada por las citadas 30.371 observaciones empresas-años para las variables $B T G_{i t}$ y $P E R M_{i t}$ y 20.360 para la variable $T I E 5 C_{i t}$.

\subsection{Caracterización de las variables dependientes: la agresi- vidad fiscal}

Un protocolo unánimemente seguido en la investigación empírica sobre impuestos consiste en combinar diferentes aproximaciones a la agresividad fiscal, ya que las estrategias tributarias de las empresas, al adoptar diferentes enfoques y modalidades, explican que no exista una única medida que capte todas las dimensiones de esta conducta. Por esta razón, la práctica habitual consiste en emplear tanto medidas basadas en la divergencia existente entre el resultado contable y la base imponible estimada, lo que ha dado en denominarse book-tax gap, como en cuantificar el tipo impositivo efectivamente soportado por las empresas. Nosotros ensayaremos con tres especificaciones alternativas de la agresividad fiscal: el book-tax gap, el tipo impositivo efectivo soportado, cuantificado con arreglo a criterios de caja, y una estimación de las diferencias discrecionales entre resultado contable y base imponible.

El book-tax gap es, como decimos, la diferencia existente 
entre una estimación de la base imponible y el resultado contable antes de impuestos, aceptándose que las prácticas fiscales son tanto mas agresivas cuanto mayor es la divergencia entre ambas magnitudes, puesto que ello, como razona Wilson (2009), estaría indicando la presencia de filiales, sucursales o establecimientos permanentes en territorios de baja tributación, generación de ingresos contables no computables fiscalmente, diferencias temporarias, etc. No obstante, Mills \& Newberry (2001) y Hanlon et al. (2005) consideran que en el segmento de las compañías no cotizadas, al que pertenecen las empresas de nuestra muestra, es posible que exista una mayor convergencia entre resultado contable y base imponible, como estrategia de protección frente a los riesgos fiscales y evitar de este modo verse implicadas en procedimientos de comprobación e inspección tributarios.

A su vez existen dos versiones del book-tax gap; la primera es la formulada por Manzon \& Plesko (2002), que deflactan esta magnitud -diferencia entre base imponible estimada y resultado contable antes de impuestos- por el valor contable del activo total; la segunda, la propuesta por Hanlon et al. (2012), que emplean el book-tax gap en forma logarítmica y en valores absolutos. Nosotros vamos a adoptar esta segunda versión, ya que, en nuestra opinión, al no deflactar el valor resultante evitamos algunos de los problemas derivados de tomar el activo contable como medida del tamaño, tales como la heterogeneidad de criterios valorativos en las diferentes partidas que lo integran o el impacto de la diversidad de políticas de amortizaciones y deterioros seguidas por las empresas ${ }^{2}$. Por tanto, nuestra primera medida de agresividad fiscal, que denominaremos $B T G_{i t}$, es la siguiente:

$$
B T G_{i t}=\operatorname{Ln} R C A I_{i t}-B I_{i t},
$$

Donde $R C A I_{i t}$ es el resultado contable antes de impuestos y $B I_{i t}$ es la estimación de la base imponible del ejercicio, obtenida como el cociente entre el gasto devengado por Impuesto sobre Sociedades y el tipo impositivo aplicable a las empresas de nuestra muestra en cada ejercicio ${ }^{3}$. Como razonan Comprix et al. (2011), Hanlon (2005), Hanlon et al. (2012) y Watrin et al. (2014), su expresión en valores absolutos nos permite apreciar la dimensión correcta de la divergencia entre ambas magnitudes, de tal suerte que si el coeficiente $\beta$ asociado a la variable experimental $B 4_{i t}$ resulta ser positivo y significativo cuando en el modelo (1) se toma $B T G_{i t}$ como variable dependiente, se rechazaría la hipótesis nula y se confirmaría la hipótesis alternativa, es decir, estaría indicando que la contratación de una Big Four estimularía las actividades de planificación fiscal de su cliente. En caso de que $\beta$ fuera negativa y significativa, dejaría constancia de que las Big Four moderarían la agresividad tributaria de éste.

La segunda de las medidas de planificación fiscal es el tipo efectivo del impuesto pagado, empleado por primera vez por Dyreng et al. (2008) y posteriormente en numerosos estudios (entre otros muchos, Armstrong et al., 2015; Chen et al., 2010; Dyreng et al., 2010; Frank et al., 2009) que se define como el cociente entre el Impuesto sobre Sociedades satisfecho, ajustado por el importe de los ingresos procedentes de los dividendos, y el resultado contable antes de impuestos. Hemos efectuado este ajuste del dividendo percibido en aquellas empresas de la muestra en las que en el activo de su balance se consignan inversiones en empresas del grupo, mul-

${ }^{2}$ En Blouin (2014), Hanlon (2003), Frischmann et al. (2008) y Manzon \& Plesko (2002), se describen con detalle los problemas asociados al book-tax gap, que provienen tanto de los errores de estimación de la base imponible como del empleo del activo total como deflactor.

${ }^{3}$ Los tipos estatutarios fueron el $30 \%$ entre 2008 y 2014, el $28 \%$ en 2015 y el $25 \%$ en 2016 y 2017. tigrupo y asociadas ${ }^{4}$, ya que conceptualmente, la deducción por doble imposición sobre dividendos debe considerarse como un importe adicional del impuesto satisfecho, aproximándose de este modo a la cuota íntegra minorada en el importe de deducciones y bonificaciones fiscales ${ }^{5}$. De obviarse este ajuste, estaríamos infraestimando la presión fiscal soportada y confundiendo la deducción practicada --que es simplemente una corrección técnica-- con prácticas fiscales agresivas, puesto que resulta evidente que los dividendos están sometidos a tributación, aunque ello tenga lugar en sede de la participada.

Hemos optado por considerar el impuesto efectivamente satisfecho, en lugar del impuesto devengado, porque entendemos que esta medida es metodológicamente más apropiada, y ello porque, a diferencia del impuesto devengado, en el impuesto pagado no inciden los ajustes por devengo, que podrían estar afectados por prácticas de dirección de resultados.

Sin embargo, desde la publicación del trabajo de Dyreng et al. (2008), la práctica habitual en la investigación sobre impuestos consiste en emplear una medida plurianual de la presión fiscal, ya que tomar el tipo efectivo de un único ejercicio puede inducir a error, por el hecho de sobreestimarla o infraestimarla, debido a la incidencia de circunstancias no recurrentes que se hayan manifestado ocasionalmente en un único ejercicio, lo que trasladaría una percepción equivocada de la actitud permanente de la empresa ante los impuestos. La propuesta plurianual de Dyreng et al. (2008), que notaremos por $T I E 5 C_{i t}$, ha sido empleada en numerosos estudios empíricos, y la medida del tipo impositivo a largo plazo que emplearemos será el cociente entre la suma del Impuesto sobre Sociedades efectivamente satisfecho en el ejercicio corriente y en los cuatro anteriores, ajustado por dividendos, y la suma del resultado antes de impuestos del ejercicio en curso y los cuatro precedentes:

$$
\operatorname{TIE} C_{i t}=\frac{\sum_{t=1}^{5}\left(I P_{i t}+\tau D_{i t}\right)}{\sum_{t=1}^{5} R C A I_{i t}},
$$

Donde $I P_{i t}$ y $D_{i t}$ son los impuestos pagados y los dividendos cobrados, respectivamente, tomados del estado de flujos de efectivo, y es el tipo impositivo estatutario. Como razonan Dyreng et al. (2008), esta opción resulta más apropiada que el promedio de los tipos efectivos de varios ejercicios, que tiende a sobreponderar el efecto de periodos de liquidación en los que el tipo efectivo pueda ser inusualmente elevado o reducido. Si en nuestro posterior análisis empírico el coeficiente $\beta$ vinculado a la variable experimental $B 4_{i t}$ resulta ser negativo y significativo cuando en el modelo (1) se toma TIE5C $C_{i t}$ como variable dependiente, se rechazaría la hipótesis nula y pondría de manifiesto que el hecho de contar con una Big Four como auditor contribuiría a reducir la carga tributaria soportada, minorando el pago de impuestos, y si $\beta$ resultase ser positiva y significativa, indicaría que la presencia de las Big Four mitigarían las estrategias fiscales agresivas.

\footnotetext{
${ }^{4}$ Dado que también pueden figurar en la cuenta de pérdidas y ganancias dividendos procedentes de otras inversiones financieras en instrumentos de patrimonio (por ejemplo, de carácter temporal y estar registradas como activos corrientes) a las que no sea de aplicación la deducción por doble imposición intersocietaria.

${ }^{5}$ La eliminación del impuesto subyacente (underlying tax) de los dividendos ha atravesado diferentes etapas a lo largo de los diez años que comprende nuestra muestra (2008-2017); así, entre 2008 y 2014 se aplicó la deducción por doble imposición contemplada en el Real Decreto Legislativo 4/2004; en 2015 el mecanismo de exención previsto en la Ley 27/2014, y en 2016 y 2017, por aplicación del Real Decreto Ley 3/2016, la limitación de dicho mecanismo al $50 \%$ de la cuota íntegra.
} 
La tercera de las medidas es la propuesta y desarrollada por Frank et al. (2009), que llevan a cabo la regresión de un modelo en el que la variable dependiente está constituida por una estimación de las diferencias entre resultado contable y base imponible, esto es, el book-tax gap, mientras que las variables independientes son las diferencias permanentes de mayor importancia contempladas en la normativa fiscal norteamericana. Los residuos obtenidos en la regresión del modelo vienen a ser la estimación de las diferencias entre resultado contable y base imponible que traen causa en las estrategias de planificación fiscal, y que, en consecuencia, son discrecionales.

En nuestro caso, el modelo para estimar las diferencias discrecionales, adaptado a la normativa española del impuesto, tiene la expresión siguiente:

$\frac{R C A I_{i t}-B I_{i t}}{A T_{i t}}=\delta_{0}+\delta_{1} \frac{I N T_{i t}}{A T_{i t}}+\delta_{2} \frac{D I V_{i t}}{A T_{i t}}+\delta_{3} \frac{P E_{i t}}{A T_{i t}}+\delta_{4} \frac{B T G_{i t-1}}{A T_{i t}}+\varepsilon_{i t}$

En el que las variables independientes captan aquellos determinantes potenciales del book-tax gap que no obedecen a estrategias de planificación fiscal y que, en cualquier caso, constituyen diferencias permanentes en las empresas españolas: $I N T_{i t}$ es el valor contable de los intangibles; $D I V_{i t}$ son los dividendos percibidos de empresas del grupo y asociadas, cuya doble imposición es eliminada, $P E_{i t}$ son los resultados imputados por el método de puesta en equivalencia, que, como en el caso de las empresas del grupo y asociadas, ya han tributado en sede de la entidad participada, y $B T G_{i t-1}$ es el book-tax gap del ejercicio precedente, para controlar, como en Frank et al. (2009), las diferencias permanentes no discrecionales que son persistentes en el tiempo (por ejemplo, ingresos procedentes de la propiedad industrial). Todas las variables están deflactadas por el valor contable del activo total al cierre del ejercicio.

Como decimos, los residuos de la regresión, $\varepsilon_{i t}$, representan nuestra estimación de las diferencias discrecionales, que notaremos como DISCR $R_{i t}$ para su incorporación al modelo (1), en el que si $\beta$, el coeficiente de la variable experimental $B 4_{i t}$, es positivo y significativo, nos llevaría a rechazar la hipótesis nula y a aceptar la alternativa, lo que permitiría concluir que las Big Four incentivan las prácticas orientadas a la reducción de la carga fiscal, y, a sensu contrario, de ser $\beta$ negativo y significativo, las Big Four limitarían las estrategias agresivas.

\subsection{Incorporación de las variables de control}

Con relación a las restantes variables independientes que anunciábamos en el modelo genérico (1), someteremos a control las características corporativas que la literatura ha venido documentando como potenciales determinantes o inhibidores de la agresividad fiscal, bien sintetizados en los trabajos de Graham et al. (2012), Hanlon \& Heitzman (2010) y Shackelford \& Shevlin (2001). Se tratan de atributos idiosincrásicos que, como indican Dyreng et al. (2017), podrían afectar a nuestros resultados, habida cuenta de que a lo largo de los diez ejercicios que integran nuestra muestra de empresas, éstas podrían variar su práctica tributaria, o bien, como demuestran Vegh \& Vuletin (2015), que las modificaciones en el ciclo económico induzcan cambios en las políticas fiscales que afectarían a la agresividad fiscal de las compañías. Para ello consideraremos el tamaño, el nivel de endeudamiento, la intensidad de intangibles, la rentabilidad económica y el riesgo fiscal, que son variables convencionalmente asociadas a las estrategias de planificación fiscal. Adicionalmente controlaremos la posición que mantienen las empresas en activos

\section{y pasivos por impuestos diferidos.}

En cuanto al tamaño, la literatura empírica aporta resultados contradictorios, ya que aunque las empresas más grandes tienen más disponibilidad de medios para desplegar estrategias eficientes de planificación fiscal (Dyreng et al., 2008), su mayor dimensión las hace más visibles, y de aquí que, como indican Dyreng et al. (2016) y Guenther et al. (2019), limiten su agresividad para no incurrir en riesgos reputacionales. No obstante, hay que tener en cuenta que las empresas de nuestra muestra, no cotizadas, tienen un reducido nivel de visibilidad, por lo que podría suceder que la protección de la reputación no constituyese en nuestro concreto caso un elemento mitigante de las prácticas más agresivas.

Con respecto al endeudamiento, la teoría de la estructura de capital predice que los incentivos para financiar las inversiones con deuda aumentan con el tipo impositivo marginal, debido a la deducibilidad fiscal de los intereses, y de aquí que sea razonable establecer una relación directa entre endeudamiento y agresividad fiscal, o inversa entre nivel de deuda y carga tributaria soportada, como documentan los trabajos de Richardson et al. (2013) y Badertscher et al. (2013). No obstante, Graham (2003) sostiene que el deseo de evitar el pago de impuestos podría ser un determinante de segundo orden en la selección de la estructura de capital en compañías con elevados niveles de deuda, como han documentado Monterrey \& Sánchez-Segura (2009) para el caso de las empresas españolas.

Nuestro siguiente control es la rentabilidad, puesto que cabe prever que las firmas más rentables tengan mayores incentivos para desplegar estrategias de planificación fiscal. La literatura documenta esta relación directa de manera unánime; entre otros muchos, los trabajos de Armstrong et al. (2015), Badertscher et al. (2013), Wilson (2009) y en España, Fernández Rodríguez (2004a).

La intensidad de intangibles explicaría un mayor gap entre resultado contable y base imponible y tipos efectivos más reducidos, por tratarse, en nuestra opinión, del incentivo más potente del sistema fiscal español. De no controlarse la presencia de intangibles, podría confundirse una presión fiscal más moderada y una mayor divergencia entre resultado contable y base imponible con prácticas tributarias agresivas. $\mathrm{Y}$ en cuanto al riesgo fiscal, su presencia moderaría la agresividad de la conducta tributaria de las empresas, empleándose como subrogado su posición de liquidez, ya que, como documentan Hanlon et al. (2013), el mantenimiento de niveles sustanciales de tesorería constituye una protección frente a los riesgos de litigios.

También someteremos a control los activos y pasivos por impuestos diferidos. En cuanto a los primeros, son la expresión contable de los créditos fiscales pendientes de aprovechamiento, como las bases imponibles negativas o las deducciones y bonificaciones fiscales no consumidas en ejercicios anteriores por insuficiencia de cuota líquida, así como las diferencias temporarias pendientes de reversión que reducirán la carga tributaria. En consecuencia, cabe sostener que las empresas que cuenten con importes significativos de activos por impuestos diferidos también mostrarán una menor propensión a implementar estrategias de planificación fiscal, ya que en estas circunstancias de tax exhaustion o saturación fiscal no serían necesarias. Y en cuanto a los pasivos por impuestos diferidos, al tratarse de partidas que aumentarán las bases imponibles futuras, cabe pronosticar la conducta contraria, esto es, que su presencia estimulará las prácticas de planificación fiscal.

Adicionalmente, incorporaremos al estudio el posible efecto que el nivel de honorarios podría ejercer sobre la actitud 
del auditor frente a la planificación fiscal. Como han razonado y documentado Donohoe \& Kneckel (2014) para el caso norteamericano, las compañías que muestran mayores niveles de agresividad fiscal abonan a sus auditores honorarios más elevados que las que desarrollan prácticas fiscales más conservadoras, hecho que, según estos autores, constituiría una manifestación de que esta mayor agresividad da lugar a una mayor exposición al auditor al riesgo de litigios y a incurrir en mayores niveles de esfuerzo en su tarea de verificación.

Por último, controlaremos en qué medida la localización de las empresas de nuestra muestra podría afectar al mayor o menor grado de agresividad fiscal, ya que las circunstancias tributarias no son homogéneas en todas las Comunidades Autónomas. Así, el nivel de enforcement podría ser diferente en los terrriorios forales (Navarra y País Vasco), ya que la gestión e inspección tributarias están transferidas a sus respectivas Administraciones. Y asimismo, la domiciliación en Canarias podría explicar una menor necesidad de planificación fiscal de las empresas, habida cuenta de la existencia de importantes estímulos y ventajas fiscales inherentes a la insularidad.

\subsection{Especificación econométrica y construcción de variables}

De conformidad con lo expuesto y argumentado en las secciones anteriores, el modelo (1) adoptará la siguiente forma econométrica:

$$
\begin{aligned}
A F_{i t} & =\alpha+\beta_{1} B_{i t}+\beta_{2} \text { TAM }_{i t}+\beta_{3} E_{N} D_{i t}+\beta_{4} R O E_{i t}+\beta_{5} T_{E S} \\
& +\beta_{6} I_{i t} T_{i t}+\beta_{7} A I D_{i t-1}+\beta_{8} \text { PID }_{i t-1}+\beta_{9} \text { ANOMRPOS }_{i t} \\
& +\beta_{10} \text { FORAL }_{i}+\beta_{11} \text { CANARIAS }_{i}+\beta_{k} \text { SECTOR } \beta_{n} A \tilde{N} O_{t}+\varepsilon_{i t}
\end{aligned}
$$

En el modelo (2), para cada firma $i$ y ejercicio $t, A F_{i t}$ es la variable dependiente representativa de la presión fiscal, en los términos que hemos definido anteriormente; $T A M_{i t}$ es el tamaño de la empresa, expresado como logaritmo neperiano del valor contable del activo total al cierre del ejercicio; $E N D_{i t}$ es el nivel de endeudamiento, definido como el cociente entre la deuda total y el valor contable del activo total al cierre de ejercicio; $R O E_{i t}$ es la rentabilidad financiera, definida como cociente entre el resultado contable antes de impuestos y el valor contable de los fondos propios al cierre de ejercicio; $T E S_{i t}$ es el cociente entre la tesorería y el valor contable del activo total, ambos al cierre de ejercicio; $I N T_{i t}$ es el cociente entre los valores contables de los activos intangibles y del activo total, ambos al cierre de ejercicio; $A I D_{i t-1}$ es el cociente entre los activos por impuestos diferidos y el valor contable del activo total, ambos al cierre de ejercicio, y $P I D_{i t-1}$ es el cociente entre los pasivos por impuestos diferidos y el valor contable del activo total, ambos al cierre de ejercicio.

La variable $A N O R M P O S_{i t}$, mediante la cual estimaremos los honorarios anormalmente elevados por el auditor a su cliente, se obtiene de los residuos de la regresión del modelo de expectativas de honorarios de Simunic (1994), y cuya expresión es la siguiente ${ }^{6}$ :

$$
\begin{aligned}
\operatorname{LnHON}_{i t}= & \beta_{0}+\beta_{1} \text { TAM }_{i t}+\beta_{2} I N V_{i t}+\beta_{3} C C_{i t}+\beta_{4} E N D_{i t} \\
& +\beta_{5}(R O P+A M O R T)_{i t}+\beta_{7} \text { OPINION }_{i t}+v_{i t}
\end{aligned}
$$

${ }^{6}$ En su versión original, el modelo incorpora la variable binaria PERDIDAS $S_{i t}$, que toma el valor 1 cuando la empresa incurre en resultados negativos y cero en caso contrario. Dado que nuestra muestra de empresas incluye solamente compañías con resultado contable antes de impuestos positivo, hemos procedido a eliminar esta variable. siendo, para la empresa $i$ y el ejercicio $t, L_{n H O N}$ el logaritmo neperiano de los honorarios facturados por el auditor a su cliente por todos los conceptos; $T A M_{i t}$ el logaritmo neperiano del activo total; $I N V_{i t}$ el cociente entre valor contable de los inventarios y valor contable del activo total; $C C_{i t}$ el cociente entre el valor contable de las cuentas a cobrar y el valor contable del activo total; $L E V_{i t}$ el cociente entre las deudas totales y el valor contable del activo total; $(R O P+A M O R T)_{i t}$ el cociente entre la suma del resultado de las operaciones ordinarias antes de dotaciones a la amortización y el valor contable del activo total, y OPINION $_{i t}$ una variable binaria que toma el valor 1 si el informe de auditoría contiene salvedades y 0 en caso contrario. Como resultado de la regresión, los valores de la variable censurada $A N O R M P O S_{i t}$, toman el valor del residuo si es positivo, y cero en caso contrario.

Finalmente, FORAL $L_{i}$ y $C A N A R I A S_{i}$ son variables binarias que tomarán el valor 1 si la empresa de la muestra está localizada en Navarra o País Vasco y Canarias, respectivamente, y 0 en otro caso, y SECTOR y $A \tilde{N} O_{t}$ son los habituales controles por efectos fijos de la filiación sectorial de cada compañía, a un nivel de profundidad de dos dígitos de CNAE, y de los choques macroeconómicos, respectivamente.

\section{Resultados empíricos}

\subsection{Estadísticos descriptivos de la muestra}

La tabla 1 muestra los estadísticos descriptivos de las variables incorporadas al modelo (2). Como podemos apreciar, la primera de las dependientes, $B T G_{i t}$, arroja un valor promedio de 5,216 en términos logarítmicos, con una moderada dispersión de 1,062 , mientras que la segunda, $T I E 5 C_{i t}$, alcanza un valor de $24,4 \%$, lo que deja constancia de una tributación que, en promedio, es inferior al tipo impositivo estatutario, que en los ejercicios que comprende la muestra osciló entre el $25 \%$ y el $30 \%$, con una elevada desviación estándar del $14,4 \%$. La tercera de las dependientes, $D I S C R_{i t}$, al tratarse del residuo de una regresión, exhibe un valor medio de cero y una fuerte dispersión de 0,108 , hecho que también puede deducirse del amplio rango de valores que es observable en los cuartiles de esta variable.

Con relación a las variables de control, el tamaño empresarial, $T A M_{i t}$, tiene un valor promedio de 9,931, expresado en forma logarítmica, mientras que el endeudamiento, $E N D_{i t}$, arroja una media del 44,1\% y una desviación estándar del $22,4 \%$, ciertamente notable y, también, claramente identificable en los valores intercuartílicos. En cuanto a las restantes variables, la rentabilidad financiera antes de impuestos, $R O E_{i t}$, alcanza una media del $20,4 \%$, lo que parece razonable teniendo en cuenta que la muestra de trabajo está configurada solamente por compañías con resultados positivos; la posición de tesorería, $T E S_{i t}$, un promedio del 13,0\% sobre el activo total; el peso relativo de los intangibles, $I N T_{i t}$, en promedio alcanza el 2,5\% del total del activo, y los activos y pasivos por impuestos diferidos del ejercicio precedente, $A I D_{i t-1}$ y $P I D_{i t-1}$, respectivamente, exhiben valores reducidos que indican su escasa importancia relativa en términos de balance, si bien este hecho no tiene porqué condicionar su impacto sobre las estrategias de planificación fiscal, como más adelante veremos.

\subsection{Correlaciones entre variables}

La tabla 2 recoge las correlaciones entre variables; sobre la diagonal están situadas las paramétricas de Pearson y bajo 
Tabla 1. Estadísticos descriptivos

La muestra está integrada por 30.371 observaciones empresas-años de sociedades mercantiles domiciliadas en España, activas, auditadas, que formulan cuentas anuales normales individuales y pertenecen a todos los sectores económicos excepto a los identificados como CNAE 64, 65, 84 y 99, con datos completos en, al menos, uno de los ejercicios comprendidos entre 2009 y 2017 y sin registrar resultados negativos de ejercicios anteriores. Al requerir que la variable TIE5C muestre valores entre 0 y 1 , la muestra quedan configurada por 30.306 observaciones para el análisis empírico cuando se emplea esta variables. Para cada observación de la empresa $i$ y ejercicio $t, B T G_{i t}$ es el book - tax gap, definido como logaritmo neperiano del valor absoluto de la diferencia entre resultado contable antes de impuestos y la base imponible estimada, obtenida como cociente entre el gasto por Impuesto sobre Sociedades y tipo impositivo estatutario; $T I E 5 C_{i t}$ es el tipo impositivo efectivo del ejercicio corriente y los cuatro precedentes, definido como cociente entre el impuesto sobre beneficios pagado, ajustado por dividendos, y el resultado contable antes de impuestos; DISCR it son las diferencias discrecionales, obtenidas como residuos de la regresión de las diferencias permanentes innatas sobre BTGit; $T A M_{i t}$ es el tamaño de la empresa, expresado como logaritmo neperiano del valor contable del activo total al cierre del ejercicio; $E N D_{i t}$ es el nivel de endeudamiento, definido como el cociente entre la deuda total y el valor contable del activo total al cierre de ejercicio; $R O E_{i t}$ es la rentabilidad financiera, definida como cociente entre el resultado contable antes de impuestos y el valor contable de los fondos propios al cierre de ejercicio; $T E S_{i t}$ es el cociente entre la tesorería y el valor contable del activo total, ambos al cierre de ejercicio; $I N T_{i t}$ es el cociente entre los valores contables de los activos intangibles y del activo total, ambos al cierre de ejercicio; $A I D_{i t-1}$ es el cociente entre los activos por impuestos diferidos y el valor contable del activo total, ambos al cierre de ejercicio; $P I D D_{i t-1}$ es el cociente entre los pasivos por impuestos diferidos y el valor contable del activo total, ambos al cierre de ejercicio; ANORMPOS it se obtiene de los residuos de la regresión del modelo de expectativas de honorarios de Simunic (1994); FORAL y 0 en otro caso, y $S E C T O R$ y $A \tilde{N} O_{t}$ son los habituales controles por efectos fijos de la filiación sectorial de cada compañía, a un nivel de profundidad de dos dígitos de CNAE, y de los choques macroeconómicos, respectivamente.

\begin{tabular}{|c|c|c|c|c|c|}
\hline Variables & Media & Desv. estándar & $25 \%$ & $\begin{array}{c}\text { Cuartiles } \\
50 \% \text { (mediana) }\end{array}$ & $75 \%$ \\
\hline BTG $_{\text {it }}$ & 5,216 & 1,062 & 3,291 & 5,120 & 6,188 \\
\hline TIE5C & $24,4 \%$ & $14,4 \%$ & $20,6 \%$ & $25,9 \%$ & $29,1 \%$ \\
\hline DISCR $_{\text {it }}$ & 0,000 & 0,108 & $-0,057$ & $-0,023$ & 0,025 \\
\hline B4 ${ }_{i t}$ & 0,250 & 0,433 & 0,000 & 0,000 & 0,500 \\
\hline TAM $_{\text {it }}$ & 9,931 & 1,195 & 9,135 & 9,717 & 10,498 \\
\hline END $_{\text {it }}$ & $44,1 \%$ & $22,4 \%$ & $25,3 \%$ & $44,1 \%$ & $61,1 \%$ \\
\hline $\mathrm{ROE}_{\mathrm{it}}$ & $20,4 \%$ & $32,7 \%$ & $8,6 \%$ & $15,3 \%$ & $25,9 \%$ \\
\hline $\mathrm{TES}_{\mathrm{it}}$ & $13,0 \%$ & $14,0 \%$ & $2,1 \%$ & $8,4 \%$ & $19,8 \%$ \\
\hline $\mathrm{INT}_{\text {it }}$ & $2,5 \%$ & $9,5 \%$ & $0,0 \%$ & $0,7 \%$ & $1,2 \%$ \\
\hline $\mathrm{AID}_{\mathrm{it}-1}$ & $0,5 \%$ & $1,1 \%$ & $0,0 \%$ & $0,2 \%$ & $0,6 \%$ \\
\hline PID $_{\text {it-1 }}$ & $0,6 \%$ & $1,3 \%$ & $0,0 \%$ & $0,1 \%$ & $0,6 \%$ \\
\hline ANORMPOS $_{\text {it- } 1}$ & 0,892 & 11,613 & 0,000 & 0,000 & 0,314 \\
\hline FORAL $_{\mathrm{i}}$ & 0,047 & 0,212 & 0,000 & 0,000 & 0,000 \\
\hline CANARIAS $_{\mathrm{i}}$ & 0,019 & 0,137 & 0,000 & 0,000 & 0,000 \\
\hline
\end{tabular}

Tabla 2. Correlaciones entre variables

Características de la muestra y definición de variables en la tabla 1. Las correlaciones por rangos de Spearman se muestran en la parte superior de la diagonal y las de Pearson en la parte inferior, y están expresadas en porcentajes. Los valores $p$ de los coeficientes de correlación se ofrecen en cursiva.

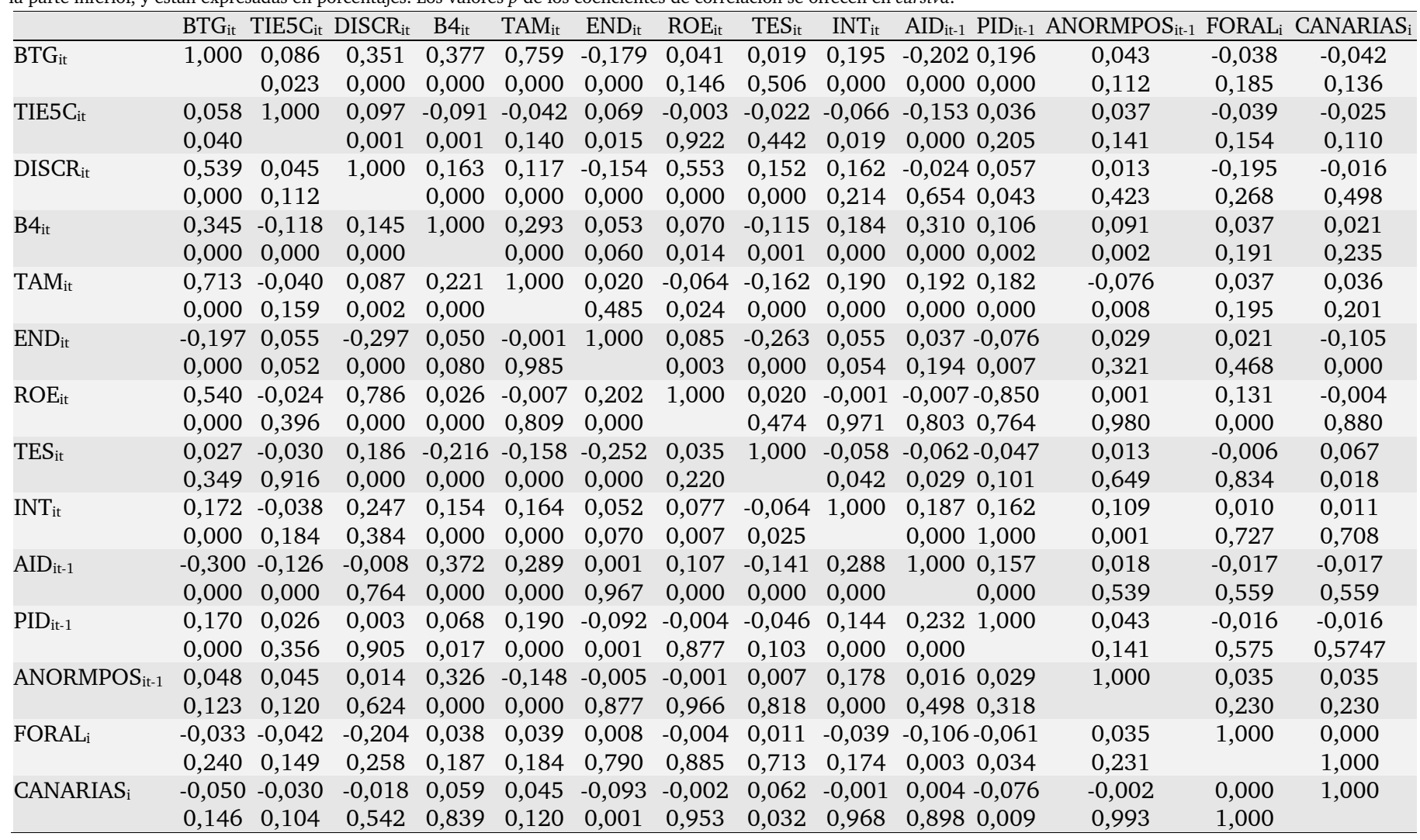

la misma las correlaciones por rangos de Spearman. Centrándonos en las más elevadas, destacan las de $B T G_{i t}$ con $D I S C R_{i t}$, $B 4_{i t}$ y $T A M_{i t}$, todas de signo positivo, lo que indica la existencia de una asociación directa del book-tax-gap con las dife- rencias discrecionales, la presencia de un auditor de calidad y el tamaño, y asimismo con $A I D_{i t-1}$, si bien en este caso de signo negativo, lo que pone de relieve cómo la presencia de activos fiscales diferidos pendientes de aprovechamiento dan 
lugar a una reducción del book-tax gap.

También exhiben una apreciable correlación las de $D_{I S C R_{i t}}$ con $R O E_{i t}$, lo que pone de manifiesto cómo las compañías más rentables tienen a generar mayores diferencias discrecionales, y la de $E N D_{i t}$ con $T E S_{i t}$, esta última de signo negativo, dejando así constancia de cómo las empresas más endeudadas muestran menores posiciones de liquidez, como consecuencia de destinarla al servicio de la deuda.

Contempladas en su conjunto, las correlaciones ofrecen valores compatibles con las documentadas habitualmente en la literatura empírica sobre impuestos y, a tenor de los mismos, nos permiten descartar la existencia de problemas asociados a la presencia de niveles elevados de multicolinealidad.

\subsection{Resultados econométricos}

Antes de presentar los resultados obtenidos del contraste de nuestra hipótesis, creemos conveniente dar a conocer una evidencia preliminar que recogemos en la tabla 3. Tras dividir la muestra en deciles de cada una de las variables representativas de la planificación fiscal, hemos recogido en tres paneles los valores de las variables independientes obtenidos de los deciles de mayor y menor agresividad fiscal. Así, en el panel A, relativo a la variable $B T G_{i t}$, en el decil de mayor agresividad fiscal, el 55,2\% de los auditores forman parte de las Big Four, frente a solamente un $11,2 \%$ de presencia de las grandes firmas de auditoría en el decil más conservador. En el panel B, correspondiente a $T I E 5 C_{i t}$, las empresas más agresivas son auditadas por una Big Four en el 52,8 \% de los casos, y únicamente el 16,8\% en el decil más conservador. Y en cuanto a $D I S C R_{i t}$, las Big Four auditan al 48,0\% de las empresas con mayores diferencias discrecionales, porcentaje que se reduce al $18,4 \%$ en el decil más conservador. En cuanto a las variables de control, cuyas medianas también incluimos en la tabla 3, en la mayoría de ellas se producen diferencias entre deciles, siendo especialmente apreciables las existentes en el nivel de endeudamiento, $E N D_{i t}$, rentabilidad, $R O E_{i t}$, y en las posiciones de tesorería, TES $S_{i t}$.

La tabla 4 ofrece los resultados obtenidos de la regresión del modelo (2) para cada una de las tres variables dependientes seleccionadas, empleando la metodología de datos de panel, con la finalidad de someter a control la heterogeneidad inobservable de las empresas de nuestra muestra, y hemos efectuado las estimaciones por efectos fijos, al ser el procedimiento apropiado al rechazarse la hipótesis nula del test de Hausman de igualdad de coeficientes entre los modelos de efectos fijos y aleatorios.

En la primera columna recogemos los derivados de emplear el book-tax gap, BTG $G_{i t}$, como variable dependiente, y en ellos se deja constancia de cómo $B 4_{i t}$, nuestra variable experimental, es positiva y estadísticamente muy significativa, hecho que viene a rechazar la hipótesis nula enunciada y nos lleva a aceptar la hipótesis alternativa, esto es, a afirmar que la presencia de un auditor de calidad hace más propensas a las empresas a mostrar una mayor divergencia entre resultado contable y base imponible, indicativa de estrategias de planificación fiscal. Con respecto a las variables de control, han resultado ser estadísticamente significativas y con signo positivo el tamaño, $T A M_{i t}$, la rentabilidad, $R O E_{i t}$, la liquidez, $T E S_{i t}$, y el peso relativo de los activos intangibles, $I N T_{i t}$, y de aquí que sea posible colegir que las empresas de mayor dimensión, las más rentables, las que operan con mayor tesorería y las más intensivas en intangibles tienen claros incentivos para implicarse en estrategias de planificación tributaria. Asimismo, el signo negativo asociado al endeudamiento, $E N D_{i t}$, indica, como está documentado en la literatura, que las empresas con un mayor nivel de deuda tienen a asumir mayores cargas fiscales.

Por el contrario, no son estadísticamente significativas las posiciones en activos y pasivos por impuestos diferidos de los ejercicios precedentes, $A I D_{i t-1}$ y $P I D_{i t-1}$, respectivamente. Además, con base en esta evidencia tampoco es posible afirmar que un elevado nivel de honorarios facturados por servicios de auditoría, representados por $A N O R M P O S_{i t}$, genere incentivos en el auditor para cooperar en las estrategias fiscales de su cliente. En otras palabras, es el tipo de auditor (Big Four), y no el nivel de honorarios de éstos, el hecho que verdaderamente afecta a la conducta fiscal de sus clientes. Por último, las variables representativas de la localización de las empresas de la muestra en territorios forales $\left(F O R A L_{i}\right)$ o en Canarias $\left(C A N A R I A S_{i}\right)$ tampoco han resultado ser significativas.

Tabla 4. Resultados de la regresión del modelo

$$
\begin{aligned}
A F_{i t}= & \alpha+\beta_{1} B 4_{i t}+\beta_{2} \text { TAM }_{i t}+\beta_{3} E N D_{i t}+\beta_{4} R_{\text {OOE }}+\beta_{5} \text { TES }_{i t} \\
& +\beta_{6} I N T_{i t}+\beta_{7} \text { AID }_{i t-1}+\beta_{8} \text { PID }_{i t-1}+\beta_{9} \text { ANOMRPOS }_{i t} \\
& +\beta_{10} \text { FORAL }_{i}+\beta_{11} \text { CANARIAS }_{i}+\beta_{k} \text { SECTOR }+\beta_{n} A \tilde{N} O_{t}+\varepsilon_{i t}
\end{aligned}
$$

Características de la muestra y definición de las variables en la tabla $1 . A F_{i t}$ es la variable dependiente representativa de la presión fiscal, pudiendo tratarse de las variables $B T G_{i t}, T I E 5 C_{i t}$ y $D I S C R_{i t}$, también definidas en la tabla 1. La significación estadística se expresa en asteriscos, a niveles superiores al $90 \%{ }^{*}, 95 \%{ }^{* *}$ y $99 \%^{* * *}$

\begin{tabular}{|c|c|c|c|c|c|c|}
\hline & \multicolumn{2}{|c|}{ BTG $_{\text {it }}$} & \multicolumn{2}{|c|}{ TIE5C $_{\text {it }}$} & \multicolumn{2}{|c|}{ DISCR $_{\text {it }}$} \\
\hline & Coeficientes & $\mathrm{t}$ & Coeficientes & $\mathrm{t}$ & Coeficientes & $\mathrm{t}$ \\
\hline Constante & $-1,880$ & $-7,56^{\text {kwktk }}$ & $-0,316$ & $-0,12^{\text {knkth }}$ & 0,112 & $5,11^{\text {****2}}$ \\
\hline B4 $4_{\text {it }}$ & 0,581 & $8,64^{* * * i k}$ & $-0,035$ & $-3,14^{* * * k+k}$ & 0,041 & $7,79^{* *+* x}$ \\
\hline $\mathrm{TAM}_{\mathrm{it}}$ & 0,944 & $8,95^{* * * k}$ & $-0,014$ & $-0,77^{* * * * *}$ & 0,010 & $4,47^{* * * *}$ \\
\hline $\mathrm{END}_{\text {it }}$ & $-1,337$ & $-4,69^{* * * k}$ & 0,44 & $2,28^{* * *}$ & $-0,081$ & $-7,78^{* k \times * x}$ \\
\hline $\mathrm{ROE}_{\mathrm{it}}$ & 0,042 & $5,37^{* * * * *}$ & $-0,002$ & $-0,34$ & 0,174 & $4,21^{* * * *}$ \\
\hline TES $_{\text {it }}$ & 0,836 & $4,17^{k * * * k}$ & $-0,001$ & $-0,06$ & 0,078 & $3,87^{* * * * *}$ \\
\hline $\mathrm{INT}_{\text {it }}$ & 0,572 & $1,98^{k *}$ & $-0,079$ & $-1,89^{k * *}$ & 0,011 & 0,28 \\
\hline $\mathrm{AID}_{\mathrm{it}-1}$ & 1,713 & $-0,97$ & $-1,021$ & $-2,58^{k \text { ** }}$ & $-0,377$ & $-1,45$ \\
\hline PID ${ }_{\text {it-1 }}$ & 2,145 & 0,97 & 0,325 & 1,06 & 0,058 & 0,03 \\
\hline ANORMPOS $_{\text {it }-1}$ & 0,142 & 1,12 & 0,114 & 0,14 & 0,071 & 0,95 \\
\hline FORAL $L_{i}$ & $-0,043$ & $-0,74$ & $-0,063$ & $-0,83$ & $-0,014$ & $-0,86$ \\
\hline CANARIAS $_{\mathrm{i}}$ & $-0,028$ & $-0,88$ & $-0,098$ & $-0,57$ & $-0,032$ & $-0,80$ \\
\hline $\mathrm{R}^{2}$ & $65,67 \%$ & & $13,04 \%$ & & $39,44 \%$ & \\
\hline $\mathrm{F}$ & 298,03 & & 15,02 & $* * * *$ & 96,58 & ****** \\
\hline $\mathrm{N}$ & 30.371 & & 20.360 & & 30.371 & \\
\hline
\end{tabular}
de probabilidad. Errores estándar corregidos por dependencia en sección cruzada y serie temporal (Petersen 2009; Gow, Ormazábal y Taylor 2010).

\begin{tabular}{|c|c|c|c|c|c|c|c|c|}
\hline Deciles de agresividad fiscal & Proporción de B4 & TAM $_{\text {it }}$ & END $_{\text {it }}$ & $\mathrm{ROE}_{\mathrm{it}}$ & $\mathrm{TES}_{\mathrm{it}}$ & $\mathrm{INT}_{\text {it }}$ & $\mathrm{AID}_{\mathrm{it}-1}$ & $\mathrm{PID}_{\text {it-1 }}$ \\
\hline \multicolumn{9}{|l|}{ Panel A: BTGit } \\
\hline 1 (más agresivo) & $55,2 \%$ & 9,812 & $25,1 \%$ & $35,1 \%$ & $11,5 \%$ & $1,4 \%$ & $3,5 \%$ & $0,1 \%$ \\
\hline 10 (más conservador) & $11,2 \%$ & 9,641 & $63,3 \%$ & $3,3 \%$ & $5,1 \%$ & $0,3 \%$ & $2,0 \%$ & $1,0 \%$ \\
\hline \multicolumn{9}{|l|}{ Panel B: CETR } \\
\hline 1 (más agresivo) & $52,8 \%$ & 9,856 & $37,8 \%$ & $22,8 \%$ & $6,6 \%$ & $1,5 \%$ & $0,2 \%$ & $0,2 \%$ \\
\hline 10 (más conservador) & $16,8 \%$ & 9,689 & $61,8 \%$ & $8,6 \%$ & $6,5 \%$ & $0,2 \%$ & $5,0 \%$ & $1,0 \%$ \\
\hline \multicolumn{9}{|l|}{ Panel C: DISCR } \\
\hline 1 (más agresivo) & $48,0 \%$ & 0,9812 & $32,6 \%$ & $17,5 \%$ & $12,7 \%$ & $1,4 \%$ & $0,3 \%$ & $0,1 \%$ \\
\hline 10 (más conservador) & $18,4 \%$ & 9,6416 & $62,9 \%$ & $3,4 \%$ & $5,2 \%$ & $0,2 \%$ & $0,3 \%$ & $0,1 \%$ \\
\hline
\end{tabular}

Tabla 3. Proporción de auditores Big Four en los deciles superior e inferior de agresividad fiscal

Características de la muestra y definición de las variables en la tabla 1. Valores correspondientes a las medianas de $T A M_{i t}, E N D_{i t}, R O E_{i t}, T E S_{i t}, I N T_{i t}, A I D_{i t}$ y $P I D_{i t}$. 
Los resultados tomando TIE5 $C_{i t}$ como variable dependiente de la regresión del modelo (2) muestran la misma evidencia y de nuevo nos conducen a rechazar la hipótesis nula: el signo negativo y muy significativo del coeficiente asociado a la variable de tratamiento, $B 4_{i t}$, implica la existencia de una relación negativa entre la contratación de una Big Four y la tesorería aplicada al pago de impuestos, de donde se colige que la presencia de un auditor de calidad favorece las actividades de planificación fiscal, dando lugar a una menor presión fiscal, y ello de manera persistente, habida cuenta de que nuestra variable dependiente contempla un horizonte temporal de cinco años.

En cuanto a los coeficientes de las variables de control, en este caso difieren ligeramente a los comentados anteriormente, al perder $R O E_{i t}$ y $T E S_{i t}$ la significación estadística y, por el contrario, alcanzarla el volumen de activos por impuestos diferidos, $A I D_{i t-1}$, hecho que indica que el aprovechamiento de los créditos fiscales pendientes de aplicar al cierre del ejercicio precedente reduce la carga fiscal soportada. En cuanto a la tercera de las regresiones, la llevada a cabo con $D I S C R_{i t}$ como variable dependiente, de nuevo confirma el efecto positivo de las Big Four sobre la planificación fiscal, siendo los signos y significaciones estadísticas de las variables de control muy similares a los obtenidos con la variable $B T G_{i t}$.

Contemplados en su conjunto, los resultados que acabamos de documentar no dejan lugar a la duda: en el caso de las empresas españolas no cotizadas, la elección de un auditor de calidad favorece las estrategias de planificación fiscal de las compañías, que tienen a mostrar una mayor divergencia entre resultado contable y base imponible $\left(B T G_{i t}\right)$, efectúan, en términos relativos y de manera constante, menores pagos en concepto de impuesto sobre beneficios (TIE5 $C_{i t}$ ) e incurren en un volumen significativamente mayor de diferencias discrecionales $\left(D I S C R_{i t}\right)$. Todos los modelos alcanzan coeficientes de determinación razonables y acordes con los documentados en la literatura, y los valores del estadístico $F$ revelan que las ecuaciones son estadísticamente significativas en su conjunto. Asimismo, el factor de inflación o agrandamiento de la varianza vinculado a cada una de las variables independientes (no mostrados aquí), que nos permite conocer en qué medida una variable independiente es una función lineal de otras variables principales, oscila en un rango entre un mínimo de 1,02 y un máximo de 1,18, con un valor medio de 1,14 , lo que elimina cualquier sospecha de que nuestros resultados puedan verse afectados por problemas de multicolinealidad.

\section{Pruebas de robustez}

Al objeto de reforzar y profundizar en la evidencia que acabamos de presentar, en esta sección efectuaremos algunas extensiones y llevaremos a cabo pruebas empíricas complementarias. Así, en primer lugar verificaremos en qué medida otros auditores también de calidad, los denominados Second Tiers, podrían ejercer el mismo o similar efecto sobre la planificación fiscal que las Big Four; en segundo lugar, abordaremos el efecto de la posible endogeneidad de la variable de tratamiento, $B 4_{i t}$, sobre nuestros resultados, y finalmente ensayaremos con el empleo de especificaciones alternativas de las variables de control.

\subsection{Auditores "Second Tiers"}

Aunque el mercado de la auditoría está dominado a nivel mundial por las cuatro grandes firmas, no es menor cierto que junto a ellas conviven y compiten otras firmas de auditoría de menor tamaño, pero también globales y con presencia a nivel internacional, que ofertan sus servicios profesionales con unos estándares de calidad generalmente reconocidos. A este grupo de auditores se les ha dado en llamar "Second Tiers. $\mathrm{y}$, si bien sus protocolos de trabajo y su lex artis son perfectamente equiparables a los de las Big Four, mostrando, como documentan Boone et al. (2010), niveles muy similares de calidad, podríamos diferenciarlas de éstas en dos características que podrían afectan a su posición frente al cliente, como son su distinto grado de dependencia económica y, como argumentan Aguiar \& Díaz (2015), una menor preocupación por la protección de su reputación.

La tabla 5 siguiente recoge los resultados obtenidos de la regresión del modelo (2) introduciendo una variable experimental adicional, TIER $R_{i t}$, que toma el valor 1 cuando la empresa es auditada por un Second Tier y cero en caso contrario ${ }^{7}$. La evidencia obtenida en la primera de las columnas, tomando $B T G_{i t}$ como variable dependiente, indica cómo las second tiers favorecen el despliegue de estrategias de planificación fiscal, a tenor del coeficiente positivo y muy significativo asociado a $T I E R_{i t}$, si bien con una intensidad menor a la de $B 4_{i t}$ $(0,352$ frente a 0,637$)$.

\section{Tabla 5. Resultados de la regresión del modelo}

$$
\begin{aligned}
A F_{i t}= & \alpha+\beta_{1} B_{i t}+\beta_{2} \text { TIER }_{t}+\beta_{3} \text { TAM }_{i t}+\beta_{4} E_{\text {END }}+\beta_{5} \text { ROE }_{i t}+\beta_{6} \text { TES }_{i t} \\
& +\beta_{7} I N T_{i t}+\beta_{8} \text { AID }_{i t-1}+\beta_{9} \text { PID }_{i t-1}+\beta_{10} \text { ANOMRPOS }_{i t} \\
& +\beta_{11} \text { FORAL }_{i}+\beta_{12} \text { CANARIAS }_{i}+\beta_{k} \text { SECTOR }+\beta_{n} A \tilde{N} O_{t}+\varepsilon_{i t}
\end{aligned}
$$

Características de la muestra y definición de las variables en la tabla 1 , a excepción de $T I E R_{i t}$, que toma el valor 1 si la empresa es auditada por una firma secondier y 0 en caso contrario. $A F_{i t}$ es la variable dependiente representativa de la presión fiscal, pudiendo tratarse de las variables $B T G_{i t}, T I E 5 C_{i t}$ y $D I S C R_{i t}$, también definidas en la tabla 1. La significación estadística se expresa en asteriscos, a niveles superiores al

\begin{tabular}{|c|c|c|c|c|c|c|}
\hline & \multicolumn{2}{|c|}{ BTG $_{\text {it }}$} & \multicolumn{2}{|c|}{ TIE5C $_{\text {it }}$} & \multicolumn{2}{|c|}{ DISCR $_{\text {it }}$} \\
\hline & Coeficientes & $\mathrm{t}$ & Coeficientes & $\mathrm{t}$ & Coeficientes & $\mathrm{t}$ \\
\hline Constante & $-1,842$ & $-7,40^{* * * \hbar}$ & $-0,313$ & $-8,13^{\text {k*k* }}$ & 0,121 & $5,26^{k * * *}$ \\
\hline B4 it & 0,621 & $9,12^{* * * *}$ & $-0,032$ & $-3,18^{* * \hbar *}$ & 0,054 & $8,11^{* n+k}$ \\
\hline TIER $_{\text {it }}$ & 0,351 & $3,21^{\text {*****}}$ & $-0,012$ & $-0,71$ & 0,032 & $2,24^{\text {*** }}$ \\
\hline $\mathrm{TAM}_{\mathrm{it}}$ & 0,937 & $8,64^{\text {*****t}}$ & $-0,105$ & $-2,81^{\text {**** }}$ & 0,110 & $4,66^{n+*}$ \\
\hline END $_{\text {it }}$ & $-1,343$ & $-4,78^{* \ldots * \hbar}$ & 0,042 & $2,21^{* * t}$ & $-0,092$ & $-7,81^{* * * *}$ \\
\hline $\mathrm{ROE}_{\mathrm{it}}$ & 0,044 & $5,37^{\text {*k*k}}$ & $-0,006$ & $-0,48$ & 0,024 & $4,14^{* \ldots * *}$ \\
\hline TES $_{\text {it }}$ & 0,823 & $4,12^{* * * *}$ & $-0,002$ & $-0,50$ & 0,069 & $3,88^{k+\cdots *}$ \\
\hline $\mathrm{INT}_{\mathrm{it}}$ & 0,539 & $1,87^{*}$ & $-0,085$ & $-1,93^{*}$ & 0,007 & 0,41 \\
\hline AID $_{\text {it }}$ & $-0,925$ & $-0,38$ & $-1,024$ & $-2,57^{* \ldots \hbar k}$ & $-0,044$ & $-1,49$ \\
\hline PID $_{\text {it }}$ & 2,250 & 1,10 & 0,392 & 1,13 & 0,056 & 0,20 \\
\hline ANORMPOS $_{\mathrm{it}-1}$ & 0,132 & 1,08 & 0,124 & 0,22 & 0,081 & 1,01 \\
\hline FORAL $_{\mathrm{i}}$ & $-0,061$ & $-0,49$ & $-0,071$ & $-0,98$ & $-0,018$ & $-0,89$ \\
\hline CANARIAS $_{i}$ & $-0,051$ & $-0,74$ & $-0,081$ & $-0,66$ & $-0,029$ & $-0,84$ \\
\hline $\mathrm{R}^{2}$ & $66,29 \%$ & & $15,69 \%$ & & $38,67 \%$ & \\
\hline $\mathrm{F}$ & 293,33 & $* * * *$ & 15,21 & 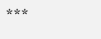 & 88,1 & 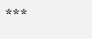 \\
\hline $\mathrm{N}$ & 30.371 & & 20.360 & & 30.371 & \\
\hline
\end{tabular}
$90 \% *$, $95 \%$ ** y $99 \% * * *$ de probabilidad. Errores estándar corregidos por dependencia en sección cruzada y serie temporal (Petersen 2009; Gow, Ormazábal y Taylor 2010).

Tomando como variable dependiente $T I E 5 C_{i t}$, los resultados, sin embargo, no corroboran el efecto favorable de su presencia sobre las prácticas de planificación fiscal, ya que $T I E R_{i t}$, si bien es de signo negativo, pierde su significación estadística a los niveles convencionales. Por último, en el modelo en el que $D I S C R_{i t}$ es la variable dependiente, $T I E R_{i t}$ vuelve a ser positivo, pero también en este caso tanto con una significación estadística como con un coeficiente inferiores a los mostrados por $B 4_{i t}$.

En definitiva, esta evidencia es sensible a la medida empírica de planificación fiscal seleccionada, de modo que los Second Tiers sí parecen influir favorablemente en términos de book-tax gap y de diferencias discrecionales --siempre en menor medida que las Big Four--, pero, a tenor de los resultados obtenidos, no puede afirmarse que contribuyan a reducir la presión fiscal en términos de impuesto efectivamente satisfecho.

${ }^{7}$ Como firmas Second Tiers hemos considerado BDO, Crowe \& Howarth, Grant Thornton, Mazars y Moore Stephens. 


\subsection{Prueba de endogeneidad}

Como complemento a los resultados que hemos venido comentando, creemos necesario plantear la posible endogeneidad de la variable experimental, $B 4_{i t}$. En efecto, y a tenor del efecto favorable que las grandes firmas de auditoría ejercen sobre la planificación fiscal, tal como hemos documentado, es verosímil que la elección del auditor pudiera estar también explicada, al menos en parte, no solamente por la calidad de su trabajo, sino también por su incidencia en las cuestiones tributarias de su cliente. De ser así, estaríamos en el caso en el que el auditor afecta a las estrategias fiscales, pero, asimismo, las estrategias fiscales también influirían en la elección del auditor, dando lugar a un problema de circularidad o simultaneidad, y por esta razón se daría que tanto la variable dependiente como la variable de tratamiento $B 4_{i t}$ estarían conjuntamente determinadas.

Para dar solución a este posible problema econométrico de endogeneidad y mitigar su impacto, el procedimiento habitual consiste en emplear regresiones en dos etapas seleccionando las oportunas variables instrumentales, ya que el empleo de regresiones lineales convencionales puede conducir a la obtención de resultados sesgados por no controlar su efecto. Con este propósito, emplearemos como instrumento en la segunda etapa los valores pronosticados de la elección de auditor surgidos de la siguiente ecuación:

$$
B 4_{i t}=\alpha+\beta_{1} T A M_{i t}+\beta_{1} E N D_{i t}+\beta_{1} R O E_{i t}+\varepsilon_{i t}
$$

En ella hemos incorporado como variables explicativas las más usuales en la literatura sobre elección de auditor (Ashbaugh \& Warfield 2003; Barton 2005; David \& Hay 2004), tamaño, endeudameinto y rentabilidad financiera. En la tabla 6 mostramos los resultados empíricos obtenidos, y en ellos podemos comprobar cómo se mantienen inalterados tanto en su signo como en la significación estadística, de manera que, tras remover el efecto de la posible endogeneidad de la variable representativa de las BigFour, es posible mantener nuestra afirmación de que la calidad del auditor afecta significativamente a la planificación fiscal de las empresas.

Tabla 6. Resultados de la regresión del modelo

$$
\begin{aligned}
& A F_{i t}=\alpha+\beta_{1} B 4_{i t}+\beta_{2} T A M_{i t}+\beta_{3} E N D_{i t}+\beta_{4} R O E_{i t}+\beta_{5} T E S_{i t} \\
& +\beta_{6} I N T_{i t}+\beta_{7} A I D_{i t-1}+\beta_{8} P_{I D} D_{i t-1}+\beta_{9} \text { ANOMRPOS }_{i t} \\
& +\beta_{10} \text { FORAL }_{i}+\beta_{11} \text { CANARIAS }_{i}+\beta_{k} \text { SECTOR }+\beta_{n} A \tilde{N} O_{t}+\varepsilon_{i t}
\end{aligned}
$$

Características de la muestra y definición de las variables en la tabla 1. Los coeficientes del modelo se estiman en dos etapas; en la segunda se emplean como instrumento los valores pronosticados de la elección de auditor obtenidos en la primera etapa. *AF it * es la variable dependiente representativa de la presión fiscal, pudiendo tratar"AF it "es la variable depending se de las variables "BTG it ", "TIE5C i " $\mathrm{t}$ y "DISCR it ", tambien definidas en la tabla 1. La significación estadística se expresa en asteriscos, a niveles superiores al $90 \%$ en sección cruzada y serie temporal (Petersen 2009; Gow, Ormazábal y Taylor 2010).

\begin{tabular}{cccrrrr}
\hline & \multicolumn{2}{c}{ BTG $_{\text {it }}$} & \multicolumn{2}{c}{ TIE5C $_{\text {it }}$} & \multicolumn{2}{c}{ DISCR $_{\text {it }}$} \\
& Coeficientes & $\mathrm{z}$ & Coeficientes & $\mathrm{z}$ & Coeficientes & $\mathrm{z}$ \\
\hline Constante & 1,624 & 1,45 & $-5,012$ & $-5,01^{* * *}$ & 0,458 & $6,01^{* * *}$ \\
B4 $_{\text {it }}$ & 5,650 & $5,82^{* * *}$ & $-0,115$ & $-2,64^{* *}$ & 0,789 & $7,68^{* * *}$ \\
TAM $_{\text {it }}$ & 0,776 & $5,99^{* * *}$ & $-0,001$ & $-0,14$ & 0,045 & $6,25^{* * *}$ \\
END $_{\text {it }}$ & $-1,388$ & $-4,30^{* * *}$ & 0,003 & $2,02^{* *}$ & 0,104 & $-2,82^{* * *}$ \\
ROE $_{\text {it }}$ & 0,021 & $1,76^{*}$ & $-0,001$ & $-0,64$ & 0,009 & $3,65^{* * *}$ \\
TES $_{\text {it }}$ & 1,181 & $3,25^{* * *}$ & $-0,024$ & $-0,79$ & 0,187 & $3,03^{* * *}$ \\
INT $_{\text {it }}$ & 1,143 & $2,03^{* *}$ & $-0,152$ & $-2,79^{* * *}$ & 0,265 & $2,98^{* * *}$ \\
AID $_{\text {it }}$ & $-4,397$ & $-3,21^{* * *}$ & $-2,458$ & $-3,98^{* * *}$ & $-3,457$ & $-5,28^{* * *}$ \\
PID $_{\text {it }}$ & 2,201 & 1,13 & 0,441 & 1,24 & 0,219 & 0,30 \\
ANORMPOS $_{\text {it-1 }}$ & 0,156 & 1,28 & 0,121 & 0,22 & 0,085 & 0,98 \\
FORAL $_{i}$ & $-0,078$ & $-0,74$ & $-0,054$ & $-0,94$ & $-0,015$ & $-0,94$ \\
CANARIAS $_{i}$ & $-0,041$ & $-0,16$ & $-0,074$ & $-0,48$ & $-0,023$ & $-0,88$ \\
\hline$R^{2}$ & $48,81 \%$ & $11,42 \%$ & $22,07 \%$ & \\
Wald $_{\text {N }}$ & $478,58^{* * *}$ & $31,45^{* * *}$ & $129,87^{* * *}$ \\
N & 30.371 & 20.360 & 30.371 & \\
\hline
\end{tabular}

No obstante, además de confirmarse nuestros resultados, la mitigación de los efectos de la endogeneidad sobre la evidencia obtenida revela un elemento adicional, como es que la variable representativa de los activos por impuestos diferidos pendientes de aprovechar, $A I D_{i t-1}$, alcanza ahora una significación estadística que no se había manifestado de una manera contundente en las pruebas empíricas anteriores. Como podemos ver, su signo, negativo en las tres especificaciones empíricas alternativas, indica que la presencia de ahorros fiscales pendientes de aplicar se erige como un notable efecto mitigador de las prácticas de planificación fiscal, ya sea reduciendo la distancia entre resultado contable y base imponible, ya sea disminuyendo el pago del impuesto, ya sea generando diferencias discrecionales.

\subsection{Especificaciones alternativas de las variables}

Nuestras últimas pruebas van encaminadas a ensayar con especificaciones alternativas de las variables de control, con el objeto de asegurar que nuestros resultados no son sensibles a este hecho. Así, hemos ensayado con el tamaño definido en términos de logaritmo de la cifra neta de negocios, con el endeudamiento expresado como cociente entre deuda a largo plazo y activo total y con la rentabilidad planteada como cociente entre resultado entes de impuestos y activo total. Por último, hemos ensayado incorporado $T I E R_{i t}$ al modelo (2) y suprimido $B 4_{i t}$, y asimismo, hemos llevado a cabo la regresión en dos etapas tomando como variable experimental $T I E R_{i t}$, pudiendo concluir que, en todos los casos, nuestros resultados, no documentados aquí, se mantienen en idénticos términos a los que hemos presentado y comentado en las secciones precedentes.

\section{Conclusiones}

Sobre una amplia muestra representativa de compañías españolas no cotizadas para el periodo comprendido entre 2009 y 2017, nuestros resultados han puesto de manifiesto que la elección de un auditor de calidad afecta de manera sensible a las prácticas fiscales de las empresas, al mostrar éstas una mayor agresividad, si bien el nivel endeudamiento y la presencia de créditos fiscales son factores que las inhiben. También hemos documentado cómo niveles elevados de honorarios del auditor no explicarían una mayor propensión de éstos a colaborar en las estrategias fiscales de su cliente. Esta evidencia estaría indicando que, en el contexto de nuestro estudio, los incentivos económicos del auditor dominarían sobre su percepción de los riesgos asociados a implicarse en las prácticas tributarias de sus clientes con la finalidad de reducir o diferir la presión fiscal soportada. Nuestros resultados son robustos y se mantienen cualitativamente en los mismos términos tras controlar el posible efecto de la endogeneidad implícita en la elección del auditor y reestimar las ecuaciones con especificaciones alternativas de variables.

Los resultados del estudio aquí presentado están en la misma línea de la corriente mayoritaria, representada por los trabajos de McGuire et al. (2012), Klassen et al. (2015), Gaaya et al. (2017) y Chen et al. (2019), que documentan la existencia de una relación estadísticamente significativa entre la calidad del auditor y la eficiencia de las prácticas de planificación fiscal de sus clientes. El trabajo de Kanagaretnam et al. (2016) es la excepción y ello, según nuestro parecer, como consecuencia de las debilidades metodológicas que hemos descrito en la sección dedicada a la revisión de la literatura. 
Consideramos que nuestro trabajo contribuye al conocimiento de las actividades de planificación fiscal de la empresa española y, en concreto, al papel del auditor como actor influyente en las mismas. Sin embargo, constituye únicamente una primera aproximación y la evidencia que hemos documentado debe tomarse con ciertas cautelas; en primer lugar, ha de tenerse muy presente que las medidas de planificación que hemos seleccionado, así como cualquiera otras que hubiéramos empleado, están basadas en estimaciones y son imperfectas, circunstancia que se reconoce en toda la literatura de manera unánime.

En segundo lugar, nuestro trabajo presenta, como sucede en los de Chen et al. (2019) y Kanagaretnam et al. (2016), la limitación derivada del hecho de desconocer, por tratarse de una tarea inabarcable, si los auditores que prestan sus servicios de verificación a las empresas de la muestra son también, de manera directa o indirecta, sus asesores tributarios, y de aquí que, de ser posible acceder a esta información, habría sido posible dar un paso más y profundizar en nuestra evidencia.

En tercer lugar, una limitación adicional proviene del hecho de no conocer la estructura de propiedad de las empresas de la muestra dada la enorme dificultad práctica que conlleva su identificación. De haber contado con esta información, nuestro trabajo también habría comprendido el análisis de los incentivos y actitudes de las distintas estructuras, con lo que nuestros resultados se habrían enriquecido signifivaticamente.

En cuarto lugar, ha de tenerse muy presente que los resultados que documentamos deben interpretarse y confinarse al estricto ámbito de las empresas que configuran nuestra muestra, es decir, a las compañías españolas no cotizadas, por lo que no deben generalizarse ni extrapolarse a otros contextos distintos, ya que la exposición al riesgo de litigios por parte del auditor, la estructura del mercado de los servicios profesionales y el entorno institucional tanto de la auditoría como de la fiscalidad podrían explicar evidencias bien distintas a las nuestras.

Finalmente, nuestro estudio debe entenderse como una primera aproximación al entendimiento del papel que el auditor puede desempeñar en un área tan crítica como la fiscalidad de su cliente y, según nuestro parecer, abre la vía hacia nuevas aportaciones que contribuyan a profundizar en nuestro conocimiento sobre la cuestión. En este sentido, consideramos interesante extender el análisis empírico a las compañías cotizadas, por tratarse de un ámbito en el que la visibilidad tanto de los auditores como de las empresas es mayor y, por consiguiente, también lo será la percepción de los riesgos por unos y otros, y de aquí que la evidencia empírica podría diferir sensiblementa a la que hemos documentado en este trabajo.

\section{Financiación}

Esta investigación no recibió ninguna subvención específica de organismos de financiación de los sectores público, comercial o sin ánimo de lucro.

\section{Conflicto de intereses}

Los autores declaran no tener nignún conflicto de intereses.

\section{Referencias}

Aguiar Díaz, I., \& Díaz Díaz, N. L. (2015). Calidad de la auditoría, Second-Tier y tamaño: su efecto en las empresas fracasadas no cotizadas españolas. Revista Española de Financiación y Contabilidad, 44(1), 24-46. https: //doi.org/10.1080/02102412.2014.982386

Altshuler, R., Grubert, H., \& Newlon, T.S. (2000). Has U.S. investment abroad become more sensitive to tax rates? En Hines Jr., J. (Ed.), International Taxation and Multinational Activity (pp. 9-38). Chicago: University of Chicago Press.

Armstrong, C., Blouin, J., Jagolinzer, J., \& Larcker, D.F. (2015). Corporate governance, incentives, and tax avoidance. Journal of Accounting and Economics, 60(1), 1-17. https://doi.org/10.1016/j.jacceco.2015.02.003

Ashbaugh, H., \& Warfield, T. (2003). Audits as a Corporate Governance mechanism: Evidence from the German market. Journal of International Accounting Research, 2(1), 1-21. https://doi.org/10.2308/jiar.2003.2.1.1

Badertscher, B., Katz, S., \& Rego, S. (2013). The separation of ownership and control and corporate tax avoidance. Journal of Accounting and Economics, 56(2-3), 228-250. https://doi.org/10.1016/j.jacceco.2013.08.005

Barton, J. (2005). Who cares about auditor reputation? Contemporary Accounting Research, 22(3), 549-586. https: //doi.org/10.1506/C27U-23K8-E1VL-20R0

Berger, P. (1993). Explicit and implicit tax effects of the R\&D tax credit. Journal of Accounting Research, 31(2), 131171. https://doi.org/10.2307/2491268

Blouin, J. (2014). Defining and Measuring Tax Planning Aggressiveness. National Tax Journal, 67(4), 875-900. https: //doi.org/10.17310/ntj.2014.4.06

Boone, J. P., Khurana, I. K., \& Raman, K. K. (2010). Do the Big 4 and the second-tier firms provide audits of similar quality? Journal of Accounting and Public Policy, 29(4), 330-352. https://doi.org/10.1016/j.jaccpubpol.2010.06. 007

Bowlin, K. O., Hobson, J.L., \& Piercey, M.D. (2015). The effects of auditor rotation, professional skepticism, and interactions with managers on audit quality. Accounting Review, 90(4), 1363-1393. https://doi.org/10.2308/ accr-51032

Chen, S., Chen, X., Cheng, Q., \& Shevlin, T. (2010). Are family firms more tax aggressive than non-family firms? Journal of Financial Economics, 95(1), 41-61. https:// doi.org/10.1016/j.jfineco.2009.02.003

Chen, J. Z., Elemes, A., \& Lobo, G. J. (2019). David versus Goliath: Audit service differentiation and audit quality in UK private firms. University of Houston. Available at SSRN: https: / / ssrn.com/abstract=3105917 or http://dx. doi.org/10.2139/ssrn.3105917

Comprix, J., Graham, R.C., \& Moore, J.A. (2011). Empirical evidence on the impact of book-tax differences on divergence of opinion among investors. Journal of the American Taxation Association, 33(1), 51-78. http://dx.doi. org/10.2308/jata.2011.33.1.51

Cook, K.A., Moser, W.J., \& Omer, T.C. (2017). Tax avoidance and ex ante cost of capital. Journal of Business Finance \& Accounting, 44(7-8), 1109-1136. https://doi.org/10. $1111 /$ jbfa. 12258

Davis, A., Guenther, D., Krull, L., \& Williams, B. (2016). Do socially responsible firms pay more taxes? Accounting Review, 91(1), 47-68. http://dx.doi.org/10.2308/ accr-51224

Davis, D., \& Hay, D. (2004). The voluntary choice of an au- 
ditor of any level of quality. Auditing, A Journal of Practice and Theory, 23(2), 37-54. http://dx.doi.org/10.2308/ aud.2004.23.2.37

DeAngelo, L.E. (1981). Auditor size and audit quality. Journal of Accounting and Economics, 3(3), 183-199. https: //doi.org/10.1016/0165-4101(81)90002-1

DeFond, M.L., \& Zhang, J. (2014). A review of archival auditing research. Journal of Accounting and Economics, 58(2-3), 275-326. https://doi.org/10.1016/j. jacceco.2014.09.002

De Simone, L., Nickerson, J., Seidman, J. K., \& Stomberg, B. (2020). How reliably do empirical tests identify tax avoidance? Contemporary Accounting Research, 37(3), 15361561. https://doi.org/10.1111/1911-3846.12573

Desai, M.A., Dyck, A., \& Zingales, L. (2007). Theft and taxes. Journal of Financial Economics, 84(3), 591-623. https:// doi.org/10.1016/j.jfineco.2006.05.005

Desai, M. A., \& Dharmapala, D. (2006). Corporate tax avoidance and high powered incentives. Journal of Financial Economics, 79(1), 145-179. https://doi.org/10.1016/j. jfineco.2005.02.002

Donohoe, M. P., \& Knechel, R. W. (2014). Does corporate tax aggressiveness influence audit pricing?. Contemporary Accounting Research, 31(1), 284-308. https://doi. org/10.1111/1911-3846.12027

Dyreng, S.D., Hanlon, M., \& Maydew, E.L. (2008). Long-run corporate tax avoidance. Accounting Review, 83(1), 6182. http://dx.doi.org/10.2308/accr.2008.83.1.61

Dyreng, S. D., Hanlon, M., \& Maydew, E. L. (2010). The effects of executives on corporate tax avoidance. Accounting Review, 85(4), 1163-1189. https://dx.doi.org/10. $2139 /$ ssrn. 1158060

Dyreng, S., Hoopes, J., \& Wilde, J. (2016). Public pressure and corporate tax behavior. Journal of Accounting Research, 54(1), 147-186. https://doi.org/10.1111/ 1475-679X.12101

Dyreng, S.D., Hanlon, M., Maydew, E.L., \& Thornock, J.R. (2017). Changes in corporate effective tax rates over the past 25 years. Journal of Financial Economics, 124(3), 441-463. https://doi.org/10.1016/j.jfineco.2017.04.001

Erickson, M., \& Wang, S. (2007). Tax benets as a source of merger premiums in acquisitions of private corporations. Accounting Review, 82(2), 359-387. http://dx.doi.org/ 10.2308/accr.2007.82.2.359

Fernández Rodríguez, E. (2004a). Los factores condicionantes de la presión fiscal empresarial española a partir de la información contable. Especial mención a las decisiones financieras. Revista Española de Financiación y Contabilidad, 33(120), 125-159. http://dx.doi.org/10.1080/ 02102412.2004 .10779516

Fernández Rodríguez, E. (2004b). La imposición efectiva de las sociedades españolas desde la óptica contable y fiscal. Madrid: Instituto de Estudios Fiscales.

Frank, M.M., Lynch, L.J., \& Rego, S.O. (2009). Tax reporting aggressiveness and its relation to aggressive financial reporting. Accounting Review, 84(2), 467-496. http: //dx.doi.org/10.2308/accr.2009.84.2.467

Frischmann, P.J., Shevlin, T., \& Wilson, R. (2008). Economic consequences of increasing the conformity in accounting for uncertain tax benefits. Journal of Accounting and Economics, 46(2-3), 261-278. https://doi.org/10.1016/ j.jacceco.2008.08.002

Gaaya, S., Lakhal, N., \& Lakhal, F. (2017). Does family ownership reduce corporate tax avoidance? The moderating effect of audit quality. Managerial Auditing Journal, 32(7), 731-744. https://doi.org/10.1108/

\section{MAJ-02-2017-1530}

Gallemore, J., \& Labro, E. (2015). The importance of the internal information environment for tax avoidance. Journal of Accounting and Economics, 60(1), 149-167. https: //doi.org/10.1016/j.jacceco.2014.09.005

Gow, I.D., Ormazábal, G., \& Taylor, D.J. (2010). Correcting for cross-sectional and time-series dependence in accounting research. Accounting Review, 85(2), 483-512. http: //dx.doi.org/10.2308/accr.2010.85.2.483

Graham, J.R. (2003). Taxes and Corporate Finance: A review. Review of Financial Studies, 16(4), 1074-1128. http://dx. doi.org/10.2139/ssrn.264516

Graham, J.R., Raedy, J.S., \& Shackelford, D.A. (2012). Research in accounting for income taxes. Journal of Accounting and Economics, 53(1-2), 412-434. https://doi.org/ 10.1016/j.jacceco.2011.11.006

Graham, J., \& Tucker, A. (2006). Tax shelters and corporate debt policy. Journal of Financial Economics, 81(3), 563594. https://doi.org/10.1016/j.jfineco.2005.09.002

Guenther, D. A., Wilson, R. J., \& Wu, K. (2019). Tax uncertainty and marginal tax avoidance. Accounting Review, 94(2), 229-247. https://doi.org/10.2308/accr-52194

Hanlon, M. (2003). What can we infer about a firm's taxable income from its financial statements? National Tax Journal, 56(4), 831-863. https://dx.doi.org/10.2139/ssrn. 419741

Hanlon, M. (2005). The persistence and pricing of earnings, accruals, and cash flows when firms have large book-tax differences. Accounting Review, 80(1), 137-166. https:// dx.doi.org/10.2139/ssrn.379140

Hanlon, M., \& Heitzman, S. (2010). A review of tax research. Journal of Accounting and Economics, 50(1-2), 127-178. https://doi.org/10.1016/j.jacceco.2010.09.002

Hanlon, M., Kelley, S., \& Shevlin, T. (2005). Evidence on the possible information loss of conforming book income and taxable income. Journal of Law and Economics, 48(2), 407-442. https://doi.org/10.1086/497525

Hanlon, M., Krishnan, G.V., \& Mills, L.F. (2012). Audit fees and book-tax differences. Journal of the American Taxation Association, 34(1), 55-86. http://dx.doi.org/10. 2308/atax-10184

Hanlon, M., Maydew, E.L., \& Saavedra, D. (2013). Understanding Why Firms Hold So Much Cash: A Tax Risk Explanation. Available at https: //www.bauer.uh.edu/departments/accy/research/ documents/Hanlon-paper.pdf

Hanlon, M., \& Slemrod, J. (2009). What does tax aggressiveness signal? Evidence from stock price reactions to news about tax shelter involvement. Journal of Public Economics, 93(1-2), 126-141. https://doi.org/10.1016/ j.jpubeco.2008.09.004

Hoopes, J.L., Mescall, D., \& Pittman, J.A. (2012). Do IRS audits deter corporate tax avoidance? Accounting Review, 87(5), 1603-1639. https://doi.org/10.1016/j. jpubeco.2008.09.004

Kanagaretnam, K., Lee, J. Lim, C.Y., \& Lobo, G.J. (2016). Relation between auditor quality and corporate tax aggressiveness: Implications of cross-country institutional differences. Auditing: A Journal of Practice and Theory, 35(4), 105-135. http://dx.doi.org/10.2308/ajpt-51417

Kim, J.B., Li, Y., \& Zhang, L. (2011). Corporate tax avoidance and stock price crash risk: Firm-level analysis. Journal of Financial Economics, 100(3), 639-662. https://doi.org/ 10.1016/j.jfineco.2010.07.007

Klassen, K. J., Lisowsky, P., \& Mescall, D. (2015). The role of auditors, non-auditors, and internal tax departments in 
corporate tax aggressiveness. Accounting Review, 91(1), 179-205. https://doi.org/10.2308/accr-51137

Klassen, K. J., Lisowsky, P., \& Mescall, D. (2017). Transfer pricing: Strategies, practices, and tax minimization. Contemporary Accounting Research, 34(1), 455-493. https: //doi.org/10.1111/1911-3846.12239

Lev, B., \& Nissim, D. (2004). Taxable income, future earnings, and equity values. Accounting Review, 79(4), 1039-1074. https://doi.org/10.2308/accr.2004.79.4.1039

Lisowsky, P. (2010). Seeking shelter: empirically modeling tax shelters using nancial statement information. Accounting Review, 85(5), 1693-1720. https://doi.org/10. 2308/accr.2010.85.5.1693

Manzon, G.B., \& Plesko, G.A. (2002). The relation between financial and tax reporting measures of income. Tax Law Review, 55, 175-214. http://dx.doi.org/10.2139/ ssrn. 264112

McGuire, S.T., Omer, T.C., \& Wang D. (2012). Tax avoidance: Do industry expert make a difference? Accounting Review, 87(3), 975-1003. https://doi.org/10.2308/accr-10215

Mills, L.F., \& Newberry, K.J. (2001). The influence of tax and non-tax costs on book-tax reporting differences: Public and private firms. Journal of The American Taxation Association, 23(1), 1-19. https://doi.org/10.2308/jata.2001. 23.1.1

Monterrey Mayoral, J., \& Sánchez Segura, A. (2009). ¿Cómo afectan los impuestos a la calidad del resultado? Evidencia empírica en las empresas españolas no cotizadas. Revista de Contabilidad, 12(1), 117-140. https://doi.org/ 10.1016/S1138-4891(09)70004-4

Monterrey Mayoral, J., \& Sánchez Segura, A. (2015). Planificación fiscal y Gobierno Corporativo en las empresas cotizadas españolas. Hacienda Pública Española, 214(3), 5589. http://dx.doi.org/10.7866/HPE-RPE.15.3.3

Myers, J.N., Myers, L.A., \& Omer, T.O. (2003). Exploring the term of the auditorclient relationship and the quality of earnings: A case for mandatory auditor rotation? Accounting Review, 78(3), 779-799. https://doi.org/10.2308/ accr.2003.78.3.779

Petersen, M. (2009). Estimating standard errors in finance panel data sets: Comparing approaches. Review of Financial Studies, 22(1), 435-480. https://doi.org/doi.org/10. 1093/rfs/hhn053

Phillips, J., Pincus, M., \& Rego, S.O. (2003). Earnings management: New evidence based on deferred tax expense. Accounting Review, 78(2), 491-521. https://dx.doi.org/ $10.2139 /$ ssrn.276997

Rezaee, Z. (2005). Causes, consequences, and deterrence of financial statement fraud. Critical Perspectives on Accounting, 16(3), 277-298. https://doi.org/10.1016/ S1045-2354(03)00072-8

Richardson, G., Taylor, G., \& Lanis, R. (2013). The impact of board of director oversight characteristics on corporate tax aggressiveness: An empirical analysis. Journal of Accounting and Public Policy, 32(3), 68-88. https://doi.org/ 10.1016/j.jaccpubpol.2013.02.004

Schrand, C.M., \& Wong, M.F. (2003). Earnings management using the valuation allowance for deferred tax assets under SFAS No. 109. Contemporary Accounting Research, 20(3), 579-611. http://dx.doi.org/10.1506/ 480D-098U-607R-5D9W

Shackelford, D.A., \& Shevlin, T. (2001). Empirical tax research in accounting. Journal of Accounting and Economics, 31(1-3), 321-387. https://doi.org/10.1016/ S0165-4101(01)00022-2

Simunic, D.A., (1994). Auditing, consulting, and auditor in- dependence. Journal of Accounting Research, 22, 679702. https://doi.org/10.2307/2490671

Vegh, C. A., \& Vuletin, G. (2015). How is tax policy conducted over the business cycle? American Economic Journal: Economic Policy, 7(3), 327-70. https://doi.org/10.1257/ pol.20120218

Watrin, C., Ebert, N., \& Thomsen, M. (2014). Book-tax conformity and earnings management: insights from European one-and two-book systems. Journal of the American Taxation Association, 36(2), 55-89. http://dx.doi.org/10. 2308/atax-50769

Wilson, R. (2009). An examination of corporate tax shelter participants. Accounting Review, 84(3), 969-999. https: //doi.org/10.2308/accr.2009.84.3.969 